\title{
Focal cerebral ischemia induces changes in oligodendrocytic tau isoforms in the damaged area
}

\author{
Mario Villa González $^{1,2}$ ｜ Laura Vallés-Saiz ${ }^{2}$ ｜ Ivó H. Hernández ${ }^{1,2,4}$ | \\ Jesús Avila ${ }^{2,4}$ | Félix Hernández ${ }^{2,3,4}$ | María José Pérez-Alvarez ${ }^{1,2,4}$ (]
}

${ }^{1}$ Departamento de Biología (Fisiología Animal), Facultad de Ciencias, Universidad Autónoma de Madrid, Madrid, Spain

${ }^{2}$ Departamento de Neuropatología Molecular CSIC-UAM, Centro de Biología Molecular

"Severo Ochoa", Madrid, Spain

${ }^{3}$ Departamento de Biología Molecular, Facultad de Ciencias, Universidad Autónoma de Madrid, Madrid, Spain

${ }^{4}$ Centro de Investigación Biomédica en Red sobre Enfermedades Neurodegenerativas (CIBERNED), Madrid, Spain

\section{Correspondence}

María José Pérez-Alvarez, Departamento de Biología (Fisiología Animal), Facultad de Ciencias, Universidad Autónoma de Madrid, C/Darwin 2, 28049 Madrid.

Email: mj.perez@uam.es

Félix Hernández, Centro de Biología Molecular "Severo Ochoa," Consejo Superior de Investigaciones Científicas, Universidad Autónoma de Madrid (CSIC-UAM), Madrid, Spain.

Email: fhernandez@cbm.csic.es

Funding information

Comunidad de Madrid, Consejería de Educación, Juventud y deporte, Comunidad de Madrid, Grant/Award Number: S2017/BMD3700; Secretaría de Estado de Investigación, Desarrollo e Innovación, Grant/Award Numbers: BFU-2008-03980,

BFU2016-77885-P; Fundación R. Areces; Centro de Investigación Biomédica en Red sobre Enfermedades Neurodegenerativas

\begin{abstract}
Ischemic stroke is a major cause of death and the first leading cause of long-term disability worldwide. The only therapeutic strategy available to date is reperfusion and not all the patients are suitable for this treatment. Blood flow blockage or reduction leads to considerable brain damage, affecting both gray and white matter. The detrimental effects of ischemia have been studied extensively in the former but not in the latter. Previous reports indicate that preservation of white matter integrity reduces deleterious effect of ischemia on the brain. Oligodendrocytes are sensitive to ischemic damage, however, some reports demonstrate that oligodendrogenesis occurs after ischemia. These glial cells have a complex cytoskeletal network, including tau, that plays a key role to proper myelination. 4R-Tau/3R-Tau, which differ in the presence/absence of Exon 10, are found in oligodendrocytes; but the precise role of each isoform is not understood. Using permanent middle cerebral artery occlusion model and immunofluorescence, we demonstrate that cerebral ischemia induces an increase in 3R-Tau versus 4R-Tau in oligodendrocytes in the damaged area. In addition, cellular distribution of Tau undergoes a change after ischemia, with some oligodendrocytic processes showing positive staining for 3R-Tau. This occurs simultaneously with the amelioration of neurological damage in ischemic rats. We propose that ischemia triggers an endogenous mechanism involving 3R-Tau, that induces colonization of the ischemic damaged area by oligodendrocytes in an attempt to myelinateinjured axons. Understanding the molecular mechanism of this phenomenon could pave the way for the design of therapeutic strategies that exploit glial cells for the treatment of ischemia.
\end{abstract}

\section{KEYWORDS}

brain, glia, ischemia, oligodendrocytes, pMCAO, tau

\section{1 | INTRODUCTION}

Most studies on brain damage after cerebral ischemia have focused on neurons. In contrast, limited attention has been paid to ischemic damage to glia. Little is known about the molecular mechanisms of ischemia on white matter (Zhang, Chopp, \& Zhang, 2013). Stroke is a leading cause of long-term disability in adults and a major cause of death worldwide (Benjamin et al., 2019). Most strokes are ischemic (80\%), triggered by a reduction or blockage of blood flow in a region of the brain area as a result of the occlusion of a cerebral artery, the middle cerebral artery (MCA) being the most prevalent in humans (Perez-Alvarez \& Wandosell, 2016). This occlusion causes severe damage to gray and white matter in the brain region most affected, called the ischemic core, which can vary in size depending on the duration 
of blood flow blockage (Mitsios et al., 2006). Brain regions that surround the ischemic core have been defined as penumbra (Dewar, Underhill, \& Goldberg, 2003; Perez-Alvarez \& Wandosell, 2016).

White matter is affected in some hypoxic-ischemic situations; however, there is limited knowledge of the pathological features of ischemia regarding oligodendrocytes (Zhang et al., 2013). In humans, some traumatic conditions cause severe damage of white matter, including occlusion of the MCA, global ischemia, brain and spinal cord trauma, and vascular dementia (Dewar et al., 2003; Matute, Domercq, \& Sánchez-Gómez, 2006; Pantoni, Garcia, \& Gutierrez, 1996).

Several studies have highlighted the sensitivity of axons and myelin cells to ischemia (del Puerto et al., 2014; Irving, Bentley, \& Parsons, 2001). Oligodendrocytes are the only cells in the central nervous system (CNS) capable of forming myelin, and such as they are crucial for the maintenance of axonal integrity and the preservation of nerve impulse under proper electrical conditions. Given that one oligodendrocyte is able to myelinated several neuronal axons, any alteration of these cells has profound consequences on functioning of CNS tract and also in gray matter (Bradl \& Lassmann, 2010). Mature oligodendrocytes are highly sensitive to ischemia, an insult that leads to reduced myelin after damage, thereby exacerbating neurological deficits (Baumann \& Pham-Dinh, 2001; Bradl \& Lassmann, 2010; Matute et al., 2006; Zhang et al., 2013). However, some reports highlight that stroke causes an increase in oligodendrocytes populations through a poorly known mechanism. It has been proposed that this effect may be involved in a brain self-repair strategy after an ischemic insult (LoPresti, Szuchet, Papasozomenos, Zinkowski, \& Binder, 1995; Zhang et al., 2013).

Myelination requires the integrity, dynamic properties, and correct spatial organization of the oligodendrocytes cytoskeleton. It is critical that oligodendrocytes microtubule networks be highly dynamic to provide the paths for organelle, mRNA, and protein trafficking through these cells, which present numerous membranous extensions (Butt \& Dinsdale, 2005). Like neurons, some microtubule properties of oligodendrocytes are regulated by microtubule associated proteins (MAPs) including the phosphoprotein tau (Migheli, Butler, Brown, \& Shelanski, 1988; Müller, Heinrich, Heck, Blohm, \& Richter-Landsberg, 1997; Richter-Landsberg, 2016). Tau is abundant in CNS, it plays an important role in neuronal morphogenesis and is essential for growth cone and motility (Liu, Lee, \& Jay, 1999). Under physiological conditions, tau plays a well-described role in microtubules stabilization. Tau is also found in mature oligodendrocytes (LoPresti et al., 1995; Müller et al., 1997).

Tau can be present in various isoforms, which are generated by alternative splicing of a single gene containing 16 exons (Andreadis, Brown, \& Kosik, 1992). Some of these isoforms are exclusive to embryonic or early development stages while others are expressed in the adult CNS. The six adult isoforms vary in the presence or absence of Exons 2, 3, and 10 (Goedert \& Jakes, 1990). Alternative splicing of tau Exon 10 gives rise to a protein with either 3 (3R-Tau) or 4 (4R-Tau) microtubule-binding domain (MBD). 3R-Tau is abundant mainly during early embryonic stages, whereas $4 R$-Tau predominates in the mature CNS, where it is found mainly in neurons (Avila, Lucas, Pérez, \& Hernandez, 2004), contributing to enhanced microtubule stability.
The functional role of tau has been widely studied in neurons but not in glia. Mature oligodendrocytes form a multibranched processes and flat membranous sheets through which to myelinate axons. These cellular structures contain a network of microtubules and microfilaments that plays a pivotal role in stabilization of myelin-forming processes (Butt \& Dinsdale, 2005). In vitro approaches with rat brain oligodendrocytes have demonstrated that tau expression is regulated during development, being more abundant during later stages. In addition, the mRNA of 3R-Tau is less abundant than that of $4 R-T a u$ in oligodendrocyte cultures. During oligodendrocyte development, 3R-Tau mRNA decreases, whereas 4R-Tau mRNA increases, as occurs in neurons (Gorath, Stahnke, Mronga, Goldbaum, \& RichterLandsberg, 2001).

To elucidate the role of 3R-Tau in oligodendrocytes after damage caused by brain ischemia, here we analyzed the pattern of 3R-Tau and 4R-Tau distribution in the brains of healthy adult rats and after cerebral ischemia. These two tau isoforms showed a similar distribution in the corpus callosum, around the nuclei of oligodendrocytes. However, 3R-Tau was also found in cellular processes. After cerebral ischemia, the levels of 3R-Tau increased in the corpus callosum and cerebral cortex of the ischemic hemisphere whereas the levels of $4 \mathrm{R}-\mathrm{Tau}$ remained similar to those found in Sham rats. Interestingly we observed a redistribution of $3 \mathrm{R}-\mathrm{Tau}$ to oligodendrocytes processes. At the same time, we observed an improvement in neurological damage in ischemic rats although the damaged area increased. We propose that cerebral ischemia induces a self-repair mechanism in the brain, mediate by $3 \mathrm{R}-\mathrm{Tau}$ induction in oligodendrocytes that have a role in order to maintain neurological functions, even if neuronal damage persists.

\section{2 | MATERIALS AND METHODS}

\section{$2.1 \mid$ Animals}

All the animals (rats and mice) were housed in the Animal Facility of the Centro de Biología Molecular "Severo Ochoa" (CBMSO) under a $12 \mathrm{hr}$ light/12 hr dark cycle in a temperature-controlled environment and were provided with food and water ad libitum. All the animal care protocols complied with national legislation (RD 53/2013) and the guidelines of the European Commission for the housing and care of laboratory animals (revised in Appendix A of the Council of Europe Convention ETS123). The Bio-Ethics Committee of CBMSO, the Spanish Research Council (CSIC), and the Consejería de Medioambiente de la Comunidad de Madrid (PROEX-412/15) approved all procedures.

Adult male Wistar rats ( 8 weeks old, 250-300 g) were obtained from the vivarium at CBMSO. We used 34 rats for this study, of which 11 were used for western blot experiments and 23 for histological staining. Animals were randomly distributed into the following experimental groups: Sham, $n=8$; permanent MCA occlusion (pMCAO)-2d, $n=3$; pMCAO5-d, $n=7$; and pMCAO-21d, $n=5$. Three tau-KO mice (B6.129S4 (Cg)-Mapt ${ }^{\text {tm1(EGFP)KIt } / J) ~ f r o m ~ J a c k s o n ~(m o u s e ~ s t r a i n ~}$ 004779) were also used to confirm the activity of tau promoter in 
interfascicular oligodendrocytes. These animals are hemizygous for an EGFP coding sequence inserted into the first exon of the microtubule-associated protein tau (Mapt) gene, thereby disrupting expression of Mapt and producing a cytoplasmic EGFP fused to the first 31 amino acids of the tau protein.

\subsection{Permanent MCA occlusion}

To occlude the origin of the MCA, a Dafilon 4-0 monofilament nylon suture (B. Braun, Tuttlingen, Germany) with its tip rounded by heating and coated with poly-L-lysine $(0.1 \% \mathrm{wt} / \mathrm{vol}$, in deionized water; Sigma, St. Louis, MO) was introduced into the right external carotid artery lumen and advanced into the internal carotid artery, until slight resistance was encountered (approximately $2.2 \mathrm{~cm}$ ). The suture was secured in place with a ligature and was maintained until sacrifice. Sham operated rats were subjected to all the surgical procedures but without suture insertion. All the animals received $200 \mathrm{mg} / \mathrm{L}$ ibuprofen (Dalsy, Abbot) in the drinking water as analgesic for $48 \mathrm{hr}$ after surgery. In addition, we applied skin anesthesia, namely lidocaine and prilocaine ( $25 \mathrm{mg} / \mathrm{g}$; EMLA, Astra Zeneca) after surgery to prevent pain. The animals were sacrificed at 2, 5, and 21 days after ischemia induction (corresponding to groups pMCAO-2d, pMCAO-5d, and pMCAO-21d).

Six hours after the induction of ischemia, rats from Sham and pMCAO-5d groups received an intraperitoneal (ip) injection of the thymidine analog 5-iodo-2'-deoxyuridine (IdU, Sigma) at a dose of $115.5 \mathrm{mg} / \mathrm{kg}$. IdU was dissolved at $10 \mathrm{mg} / \mathrm{ml}$ in a solution containing saline with $4 \mu \mathrm{l} / \mathrm{ml}$ of $10 \mathrm{~N} \mathrm{NaOH}$ as described (Merkley, Jian, Mosa, Tan, \& Wojtowicz, 2014).

\subsection{Neurological deficit score}

To ensure the correct induction of cerebral ischemia, the Neurological deficit score of each animal was measured before surgery, and $6 \mathrm{hr}$ post-pMCAO, using the method described by (Yrjänheikki et al., 2005). The test was conducted in a blind manner. We used a seven-point neuromotor function scale with values ranging from 0 to 6 as we described in (Perez-Alvarez, Mateos, Alonso, \& Wandosell, 2015) to calculate an ischemic damage index (IDI). We excluded all the pMCAO rats that did not reach an IDI of 2-3 at $6 \mathrm{hr}$ post-pMCAO. In addition, we evaluated evolution of neurological deficits at 1, 2, 5, and 21 days postsurgery. Other neurological anomalies, such as alterations in balance, reflex responses (corneal, palpebral, postural correction), and sensorial perception (acoustic, visual localization), were also tested in all the rats.

\section{4 | Tissue collection}

For western blot and RT-PCR analysis, animals were sacrificed by exposure to $\mathrm{CO}_{2}$ for 2,5 or 21 days after onset of pMCAO. Samples contain the right parietal cortex including the underlying corpus callosum (ischemic core), were dissected out and maintained at $-80^{\circ} \mathrm{C}$ for subsequent analysis.

For histological studies, we deeply anesthetized animals using thiobarbital $(60 \mathrm{mg} / \mathrm{kg}) \mathrm{ip}$, followed by a transcardially perfusion using ice-cold $0.1 \mathrm{M}$ phosphate-buffered saline (PBS) and $4 \%$ paraformaldehyde in $0.1 \mathrm{M}$ PBS, pH 7.4 (4\% PFA). After perfusion, the brains were removed from the skull and postfixed overnight at $4^{\circ} \mathrm{C}$ by immersion in 4\% PFA. Afterward, we cryoprotected brains using a sequential immersion in 10 and $30 \%$ of sucrose in PBS, at $4^{\circ} \mathrm{C}$ for 48-72 hr. Finally, brains were embedded in Tissue-Tek medium (Sakura, Zoeterwoude, the Netherlands). Coronal cryostat sections (25- $\mu \mathrm{m}$ thick) were collected onto Superfrost Ultra Plus slides (Thermo Scientific, Braunschweig, Germany), air dried at least $1 \mathrm{hr}$ at room temperature (RT) and stored at $-20^{\circ} \mathrm{C}$ until use. Sections located from -2 to +2 relative to Bregma (Paxinos \& Watson, 2007) were selected for immunohistochemistry/immunofluorescence procedures.

\subsection{Histological quantification of ischemic area}

To quantify the ischemic area, we used the Nissl staining method previously described (Mateos, Perez-Alvarez, \& Wandosell, 2016). All the histological quantification of ischemic area was conducted in a blind manner. In this regard, three consecutive brain slices of the same Bregma (from -2 to +2 ) (Paxinos \& Watson, 2007) from the animals in the different experimental groups were stained using the Nissl method with cresyl violet $(2 \%$ in acetate buffer, Sigma C1791). Stained slides were observed under light field microscope (Olympus BX63) and the images were captured at $\times 4$ magnification using an Olympus DP74 camera. The entire right hemisphere of each brain section was photographed using 15-18 pictures and assembled using the photomerge tool of Photoshop (version CC 2018). To determinate ischemic damage area, we used morphological criteria as described previously (Mateos et al., 2016), namely, the presence of cells with aberrant morphology (triangular in shape, condensation of cytoplasm and karyoplasm visualized by presence of a dark staining); parenchyma vacuoles; high density of small nucleus (belonging to reactive glial cells); low density of neuronal nuclei; tissue disorganization. The ischemic damage area was calculated using Fiji software (Schindelin et al., 2012) and expressed as percentage of damage area with respect to the total area of the hemisphere.

\subsection{Immunofluorescence and immunohistochemistry}

Brain sections were incubated with $0.1 \mathrm{M}$ glycine in PBS $1 \mathrm{X}$ to eliminate autofluorescence. The samples were treated with $10 \mathrm{mM}$ citrate buffer, $\mathrm{pH} 6$ for $30 \mathrm{~min}$ at $85^{\circ} \mathrm{C}$. After the samples were incubated in blocking buffer containing PBS with $0.1 \%$ Triton X-100, 1\% horse 
serum, $1 \% \mathrm{BSA}, \mathrm{pH}=7.4$. Brain samples were then incubated for 24-48 $\mathrm{hr}$ at $4{ }^{\circ} \mathrm{C}$ with the appropriate primary antibodies diluted in blocking solution: anti 3R-Tau at 1:500 (Millipore Cat 05-803), anti 4R-Tau (1:300, Millipore Cat 05-804); anti-Olig2 at 1:200 (Millipore, Cat AB9610). Next, they were incubated with the appropriate fluorescent secondary antibody, namely anti-rabbit Alexa 555 and antimouse Alexa 488 (1:500; ThermoFisher), for 1 hr at RT. DAPI (Merck) was used as a fluorescence nuclear stain (1:500). The samples were mounted using Fluoromount-G (Southern Biotech). In some samples, primary antibodies were removed to determine the specificity of the immunoreactions.

For immunohistochemistry staining after incubation with the primary antibody, brain sections were washed with PBS-0.1\% Triton X-100 and treated with PBS-1\% hydrogen peroxide to quench endogenous peroxidase activity. Afterward, sections were incubated $30 \mathrm{~min}$, at RT, with secondary antibody biotinylated anti-mouse IgG (1:200 in PBS - 1\% goat serum), then with avidin-biotin-peroxidase complex (VECTASTAIN-ABC kit, Vector laboratories Inc., Burligame, CA) for $30 \mathrm{~min}$. Next, they were incubated with FAST 3,3'-diaminobenzidine tablets (Sigma, D4168) until the desired intensity of stain was achieved (10-20 min). After rinsing with PBS, brain sections were first dehydrated using ethanol (70,90, and 100\%), cleared with xylol and then mounted using DPX fast mounting medium (toluene base) (PanReac-AppliChem, 255254.1608).

The immunostained slides were observed under an Olympus BX63 microscope and images captured using an Olympus DP74 camera. Confocal images were obtained using a LSM710 confocal laser-scanning microscope coupled to an inverted AxioObserver microscope (Zeiss).

\section{7 | Quantification of Olig2 ${ }^{+}$cells and 3R-Tau oligodendrocytes in brain section}

We used three serial brain slices of the same Bregma to quantify the number of Olig2 ${ }^{+}$cells and 3R-Tau ${ }^{+}$oligodendrocytes. We stained the samples using the appropriated primary antibodies and observed the slides under a fluorescence microscope. The images of four different fields were captured at 20X magnification using an Olympus DP74 camera. The number of positives cells was calculated using Fiji software (Schindelin et al., 2012) and expressed as number of positive cells in all image. We used Analyze Particles tool of Fiji (size: 55-infinity, circularity $0.33-1$ ) to count Olig2 ${ }^{+}$ cells of each field automatically. To facilitate counting all the images were previously modified by increasing contrast, using Fiji. To quantify double positive cells (Olig2 ${ }^{+} / 3 \mathrm{R}-\mathrm{Tau}^{+}$) we use the manual count tool of Fiji. We selected four regions of the ischemic hemisphere of each animal: corpus callosum, primary and secondary motor cortex M1/M2 (penumbra area at 5 days post-pMCAO transformed in ischemic core at 21 days), primary somatosensory cortex forelimb region $\mathrm{S} 1 \mathrm{fl}$ (ischemic core at 5 and 21 days postischemia) and subventricular zone (SVZ) of the lateral ventricle.

\section{8 | Western blotting}

Western blot analysis was performed as described previously (PerezAlvarez et al., 2015). Samples contains the right parietal cortex and the underlying corpus callosum of rats belonging to the Sham and pMCAO groups were homogenized in a buffer containing: $50 \mathrm{mM}$ Tris $\mathrm{pH}=8,150 \mathrm{mM} \mathrm{NaCl}, 1 \%$ Triton X-100, $5 \mathrm{mM}$ EDTA, protease inhibitor cocktail (Sigma-Aldrich) and phosphatase inhibitors (2 $\mathrm{nM}$ okadaic acid and $1 \mathrm{mM} \mathrm{Na}_{3} \mathrm{VO}_{4}$ ) for $30 \mathrm{~min}$ at $4{ }^{\circ} \mathrm{C}$. Samples were then centrifuged at $10,000 \mathrm{~g}$ for $5 \mathrm{~min}$ and the supernatants were collected for western blot analysis. The protein concentration of each sample was determined using the DC Protein Assay kit (BioRad, Hercules, CA). The proteins were resolved by SDS-PAGE (10\%) using a Mini-Protean system (BioRad) and $20 \mu \mathrm{g}$ of total protein was loaded in a buffer containing: $0.063 \mathrm{mM}$ Tris $\mathrm{pH}=6.8,10 \%$ SDS, $25 \%$ glycerol, $5 \%$ $\beta$-mercaptoethanol, and $0.5 \%$ bromophenol blue. Samples were heated at $95^{\circ} \mathrm{C}$ for $5 \mathrm{~min}$ before loading. After electrophoresis, the proteins were transferred intro PVDF membranes (Merck-Millipore, Darmstadt, Germany) for $2 \mathrm{hr}$ at $300 \mathrm{mV}$ using an electrophoretic transfer system (BioRad). The membranes were then blocked for $1 \mathrm{hr}$ with 5\% BSA in PBS containing 0.1\% Twen-20 (PBS-T) and incubated overnight at $4{ }^{\circ} \mathrm{C}$ with the primary antibodies: anti 3R-Tau (1:500, Millipore Cat 05-803), anti4R-Tau (1:500, Millipore Cat 05-804), antiGADPH (1:1,000, Santa Cruz, Clone G9, Cat 365062). Membranes were rinse three times with PBS-T and incubated for $1 \mathrm{hr}$ at RT with the secondary antibodies (Goat Anti-Rabbit IgG-HRP Cat. No. 4030-05 and Goat Anti-Mouse IgG-HRP Cat. No. 1030-05). Immunoreactivity was detected by the Western Lightning Plus ECL detection system (Perkin Elmer, Waltham, MA). The relative density of the bands was calculated from the optical density of the selected area (band) using Fiji Software. The values were normalized to GADPH levels detected in the same membrane and compared with control values (Sham). The bar graphs represent the mean \pm SEM of brain lysates obtained from each rat.

\subsection{RNA extraction and cDNA synthesis}

Total tissue RNA was extracted from right parietal somatosensory cortex including the underlying corpus callosum of Sham $(n=4)$ and MCAO rats $(n=16)$ using the Maxwell 16 LEV simplyRNA Tissue Kit (Promega, AS1280). Quantification and quality determination of RNA was done on a Nanodrop ND-1000 spectrophotometer and Nanodrop 1000 v.3.7.1 (Thermo Scientific). Retrotranscription (RT) reactions were performed using the iScript cDNA Synthesis kit (Bio-Rad, PN170-8891) following the manufacturer's instructions. In brief, $500 \mathrm{ng}$ of total RNA from each sample was combined with $5 \mu \mathrm{l}$ of master mix (which includes all necessary reagents along with a mixture of random primers and oligo-dT for priming). The reaction volume was completed up to $20 \mu \mathrm{l}$ with DNase/RNase-free distilled water (Gibco, PN 10977). Thermal conditions consisted of the following steps: $5 \mathrm{~min}$ at $25^{\circ} \mathrm{C} ; 20 \mathrm{~min}$ at $46^{\circ} \mathrm{C}$ and $1 \mathrm{~min}$ at $95^{\circ} \mathrm{C}$. 


\subsection{0 | MAPT PCR flanking primers}

Specific primers were designed to amplify $3 R$ (forward, $5^{\prime}$ gcttgtagactatttgcaccttg- $3^{\prime}$; reverse, $5^{\prime}$-tgcccatgccagacctaaag- $\left.3^{\prime}\right) 4 \mathrm{R}$ (forward, 5'-ccgggacgtgtttgatattgtc- $3^{\prime}$; reverse, $5^{\prime}$-aagctggatcttagcaacgtc$3^{\prime}$ ) and total tau (forward, 5'-gcatcgacatggtggactct-3'; reverse, 5'gatcacaaacctgcttggc- $\left.3^{\prime}\right)$. The following PCR amplification protocol was used: $3 \mathrm{~min}$ at $95^{\circ} \mathrm{C}+25$ cycles $\left(30 \mathrm{~s}\right.$ at $95^{\circ} \mathrm{C}+1 \mathrm{~min}$ at $60^{\circ} \mathrm{C}$ $+30 \mathrm{~s}$ at $\left.72^{\circ} \mathrm{C}\right)+7 \mathrm{~min}$ at $72^{\circ} \mathrm{C}$. PCR products were resolved on $3 \%$ agarose/gel green (Biotium, 41004) gels run at $125 \mathrm{~V}$ for $45 \mathrm{~min}$. Images were scanned with densitometer (Bio-Rad, GS-900) and quantified with Image Laboratory 5.2 (Bio-Rad). 3R and 4R tau isoforms were normalized by the total levels of tau and finally the $3 R / 4 R$ ratio was calculated.

\subsection{1 | Statistical analysis}

GraphPad Prism software was used for all statistical analysis. All the data are presented as the mean \pm SEM. In the IDI analysis, group effects on neurological deficit score were examined by two-way analysis of variance followed by Bonferroni's test. The data from western blot, RT-PCR, and immunofluorescence quantification were analyzed by a nonparametric Student's $t$ test. $p \leq .05$ value was considered statistically significant $\left({ }^{*} p \leq .05 ;{ }^{* *} p \leq .01\right.$ and ${ }^{* * *} p \leq .001$; $\left.{ }^{* * * *} p \leq .0001\right)$.

\section{3 | RESULTS}

\section{1 | Differential distribution pattern of 3R-Tau and $4 \mathrm{R}$-Tau in corpus callosum and cerebral cortex}

To determine the expression profile of 3R-Tau and 4R-Tau isoforms in healthy brain, we stained coronal brain sections of adult rats (from Bregma -2 to 2) using two isoform-specific antibodies. As illustrated in Figure $1 \mathrm{a}$ both antibodies dye intrafascicular oligodendrocytes in corpus callosum with a similar pattern, showing intense stain of the perinuclear cytoplasm.

(a)

Dapi

Olig2

3R/4R-Tau

Merged
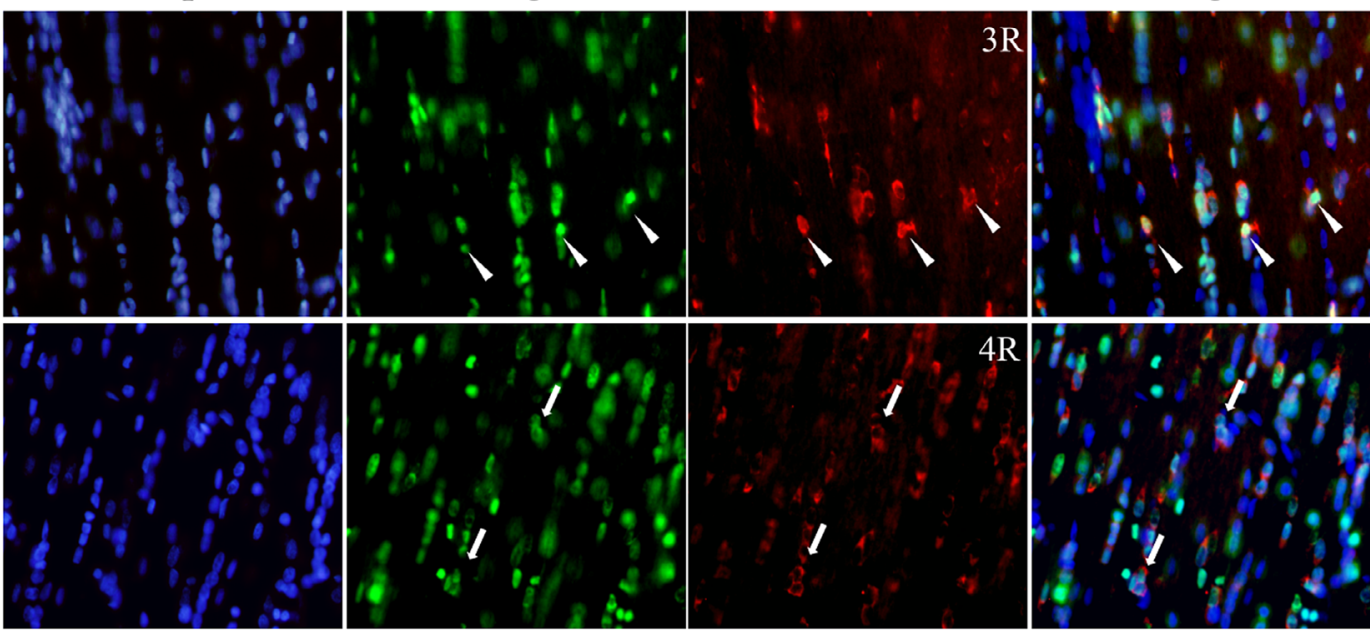

(b)
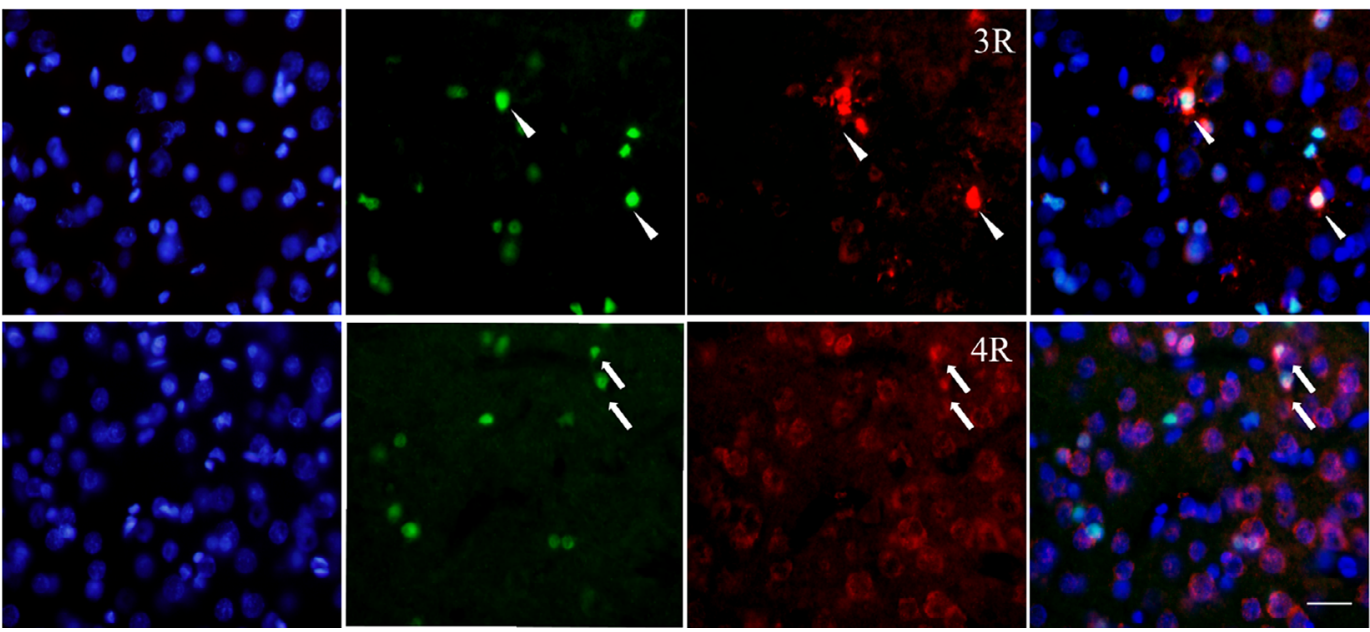

FIGURE 1 Differential pattern of 3R-Tau and 4R-Tau distribution in the corpus callosum and cerebral cortex of healthy rats.

Immunofluorescence of 3R/4R-Tau (red), Olig 2 (green) and DAPI (blue) of a representative coronal brain slide (25 $\mu \mathrm{m})$ from a healthy rats.

(a) Images corresponding to the corpus callosum and (b) cerebral cortex. All the images were captured using a fluorescence microscope. Scale bar: $25 \mu \mathrm{m}$. Arrowheads: 3R-Tau ${ }^{+}$oligodendrocytes with positive stain in their processes. Arrows: 4R-Tau ${ }^{+}$oligodendrocytes 
3R-Tau was also present in the processes of some oligodendrocytes in the corpus callosum (Figure 1a, arrowheads). In the parietal cortex, 3R-Tau staining showed a similar pattern to that of the corpus callosum, oligodendrocytes presenting clearly stained processes (Figure 1b, arrowheads). However, the distribution of $4 \mathrm{R}-\mathrm{Tau}$ in the parietal cortex was predominantly neuronal with a sparse pattern of oligodendrocytic staining (Figure 1b, arrows). In addition, immunofluorescence using an anti-Olig2 antibody in brain sections of transgenic mice expressing EGFP protein under the endogenous Tau promoter (B6.129S4(Cg)-Mapttm1(EGFP) $\mathrm{Klt} / \mathrm{J}$ ), showed that the tau promoter is highly active in interfascicular oligodendrocytes of the corpus callosum. Confocal microscopy images revealed the colocalization of EGFP with anti-Olig2. EGFP showed a cytoplasmic distribution in oligodendrocytes of the corpus callosum, mainly around nuclei (see orthogonal view, Supplementary S1) resembling the pattern observed in healthy rats when using specific antibodies against 3R-Tau and 4R-Tau.

\subsection{After pMCAO, the damaged area increased over time but the neurological score of the animal improved}

To analyze the effect of focal cerebral ischemia in 3R-Tau versus 4RTau distribution in rat brain, we induced pMCAO in rats, maintaining the suture until sacrificed at 2, 5, or 21 days postsurgery (pMCAO-2d, pMCAO-5d, pMCAO-21d groups, respectively). To confirm the correct induction of brain ischemic damage, we used two different approaches, namely: histological analysis (measuring the percentage of damage area with respect to the total surface of the injured hemisphere) and neurological score (IDI). We observed similar levels of ischemic damage at 2 and 5 days post-pMCAO, measured as percentage of damage area with respect to the total area of the hemisphere: $30.6 \pm 1.5$ and $33.8 \pm 0.02$, (Figure 2 a Sham versus pMCAO-2d and pMCAO-5d and Figure 2b). Interestingly, at 21 days post-pMCAO, the affected area increased to $48.8 \pm 2.05$ (Figure 2a pMCAO-21d and Figure $2 \mathrm{~b}$ ). We also calculated the IDI of the animals before and (a)

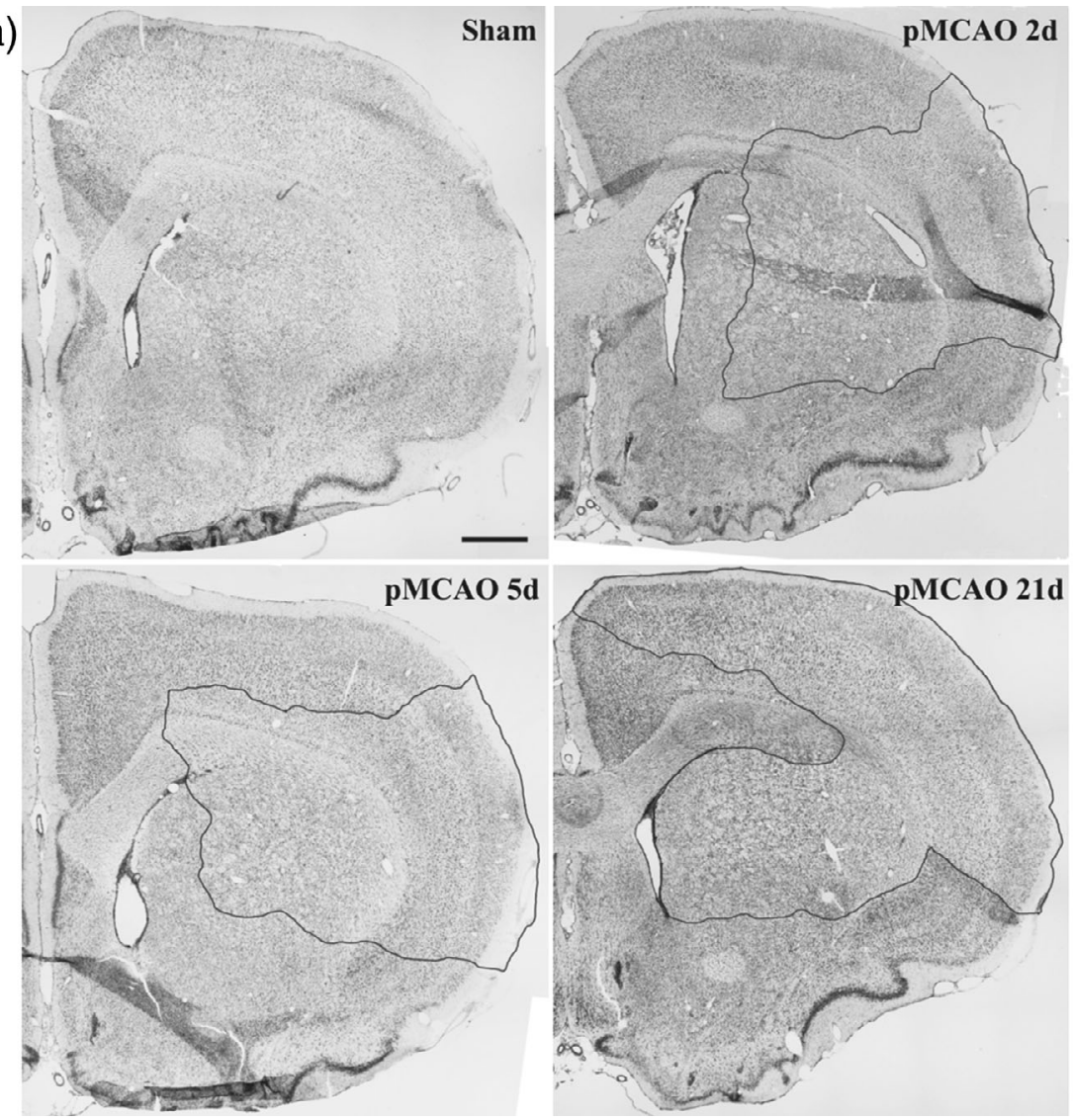

(b)

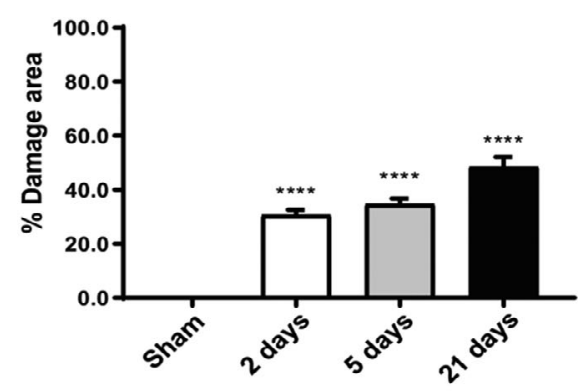

(c)

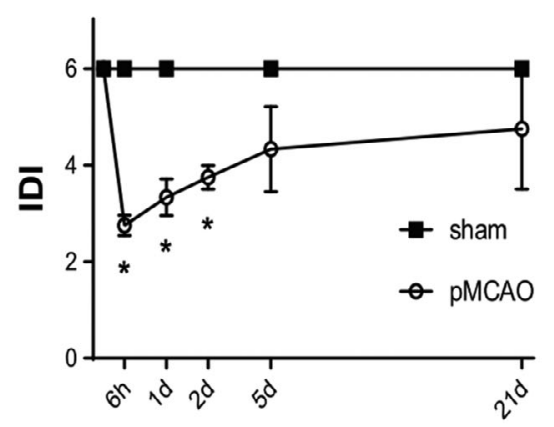

FIGURE 2 Temporal evolution of ischemic damage after permanent middle cerebral artery occlusion (pMCAO). (a) Representative montages of low magnification images of a brain hemisphere of Nissl-stained coronal brain section $(25 \mu \mathrm{m}$ ) of Sham or ischemic rats (pMCAO) 2,5 , and 21 days after ischemia induction. The solid line indicates the limit of the ischemic damage. Scale bar $=1 \mathrm{~mm}$. (b) Graph represents the percentage of damaged area with respect to the total hemisphere, measured in six consecutive coronal brain sections for each animal, using $25 \mu \mathrm{m}$ coronal slices of pMCAO rats 2,5 , and 21 days after injury. Bars represent the means $\pm S E M(n=3-4)$. (c) The ischemic damage index (IDI) was measured using a seven-point scale by a standard neurological test: 0 corresponds to the lowest and 6 to the highest neurological score. Data correspond to the mean of all rats used in each experimental group: Sham, $n=8$; pMCAO $n=15$. Analysis of variance (ANOVA) test. $p$-Values: ${ }^{*} p<.05$, 
after pMCAO (Figure 2c). Prior to surgery, all the rats reached the maximum IDI score of 6 . After $6 \mathrm{hr}$ of pMCAO induction, the score was $2.6 \pm 0.2$ and it tended to increased gradually, almost reaching the value of Sham animals $(4.8 \pm 1.3)$ at 21 days postsurgery.

These results confirm that pMCAO had a significant and consistent detrimental effect on the parietal cortex, corpus callosum, and striatum, which was reflected in significant reduction of the IDI from $6 \mathrm{hr}$ to 2 days postischemia with respect to Sham rats. Although ischemic rats recover spontaneously the neurological score of Sham group at 5-21 days post-pMCAO induction, reaching an IDI of almost 5 , the affected area continued to increase over the time.

\section{3 | pMCAO leads to an increase in 3R-Tau but not 4R-Tau levels in the corpus callosum and parietal cortex}

To analyze the effect of ischemia on 3R-Tau and 4R-Tau levels in the most injured region, namely the ischemic core, we used parietal cortex brain lysates from both hemispheres of Sham, pMCAO-2d, and pMCAO-5d rats. A significant increment in 3R-Tau on the ipsilateral hemisphere was observed with respect to Sham animals at 5 days post-pMCAO (127.25 $\pm 3.37 ; p=.0092)$ (Figure 3a,b). This increment is also observed at 21 days postischemia $(217.75 \pm 1.53 ; p \leq .001)$ (Figure Supplementary S2). However, 4R-Tau remained at the same levels as in Sham animals (Figure 3a,c). These results are in agreement with those obtained using a semiquantitative PCR approach, which showed an increase in the mRNA ratio of 3R-Tau/4R-Tau at 2 days post-pMCAO: $0.372 \pm 0.008$ versus $0.499 \pm 0.05 ; p=.01$ (Figure $3 d$ ).
We did not find any significant changes in the amount of 3R-Tau and $4 \mathrm{R}-\mathrm{Tau}$ in the contralateral hemisphere at 2 or 5 days post-pMCAO compared to Sham rats (data not shown).

To study the effect of pMCAO on the distribution pattern of the two isoforms of tau, we stained coronal brain sections of ischemic rats using isoform-specific antibodies (Figure 4). Our results demonstrated that 3R-Tau staining increased notably in the ipsilateral side of ischemic region at 5 and 21 days postsurgery (Figure 4a). Staining was especially strong in the white matter of the corpus callosum and tracts from the striatum (Figure 4a, arrows). Similar to the western blot results, immunohistochemical analysis using anti 4R-Tau antibody showed staining in cerebral cortex, corpus callosum and striatum that did not change at 5 or 21 days postischemia induction (Figure $4 b$ ).

\section{4 | 3R-Tau in oligodendrocytes after pMCAO: Analysis of regional distribution in ischemic area over time}

Our previous results indicate that cerebral ischemia induces changes in the levels of 3R-Tau but not 4R-tau in the corpus callosum and cerebral cortex. We next addressed whether the increase in 3R-Tau levels was homogeneously distributed in the damaged area or whether regional differences occurred, which could indicate the involvement of this isoform in neurological recovery processes after cerebral ischemia, a notion supported by the neurological scores. Using rat brain sections from Sham, pMCAO-5d, and pMCAO-21d animals, we studied the number of Olig2 $^{+}$and double staining Olig2 ${ }^{+} / 3 \mathrm{R}-\mathrm{Tau}^{+}$cells. In corpus callosum (Figure 5(b)), we did not find
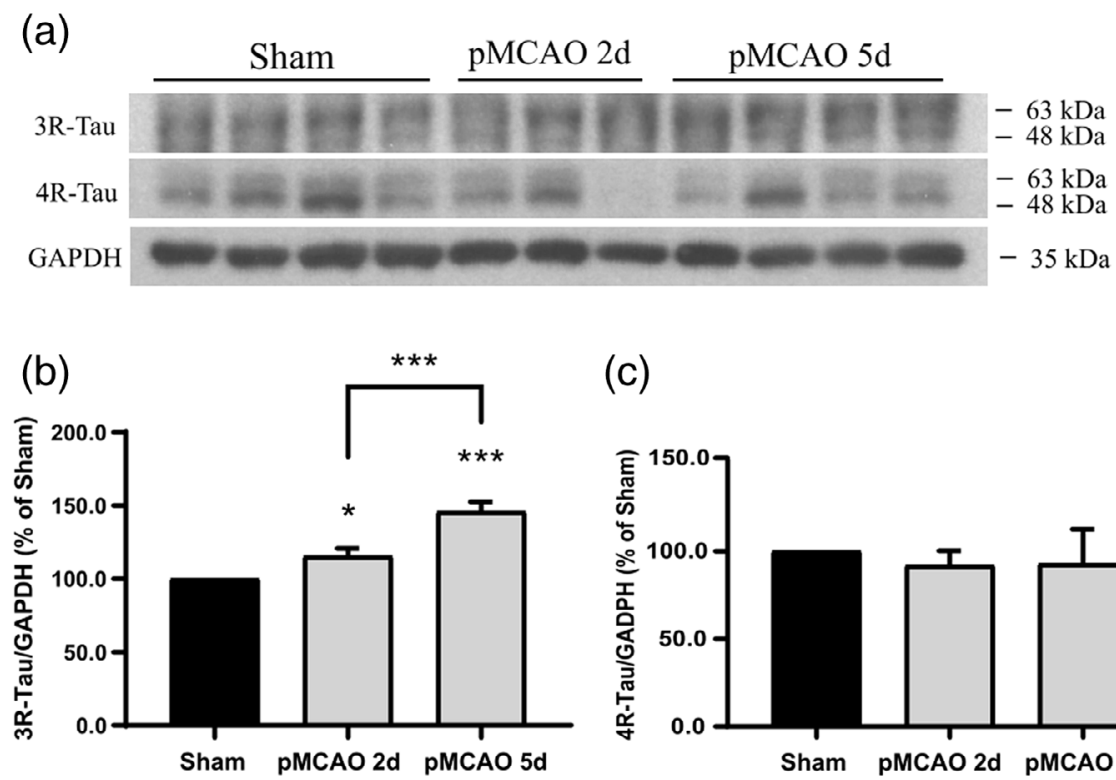

(c)

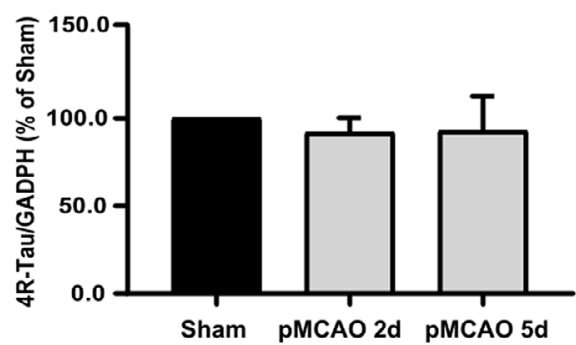

(d)

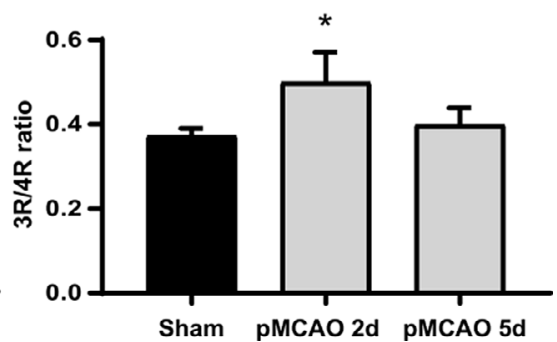

FIGURE 3 Increase in 3R-Tau levels in the ischemic core after permanent middle cerebral artery occlusion (pMCAO). (a) Western blots analysis of 3R-Tau and 4R-Tau protein levels after 2 or 5 days post pMCAO $(n=11)$. Histograms shows the abundance of 3R-Tau (b) or 4R-Tau (c) isoforms in homogenates of the parietal cortex and underlying corpus callosum of ischemic rats (pMCAO), expressed as a percentage of values of the Sham-operated rats. (d) Quantitative RT-PCR analysis of 3R and 4R mRNA isoforms. Each bar represents the mean $\pm S E M(n=3-4)$. Student's $t$ test; $p$-values: ${ }^{*} p<.01 ;{ }^{* *} p<.005 ;{ }^{* * *} p<.001$ 
(a)

$$
\text { 3R-Tau }
$$
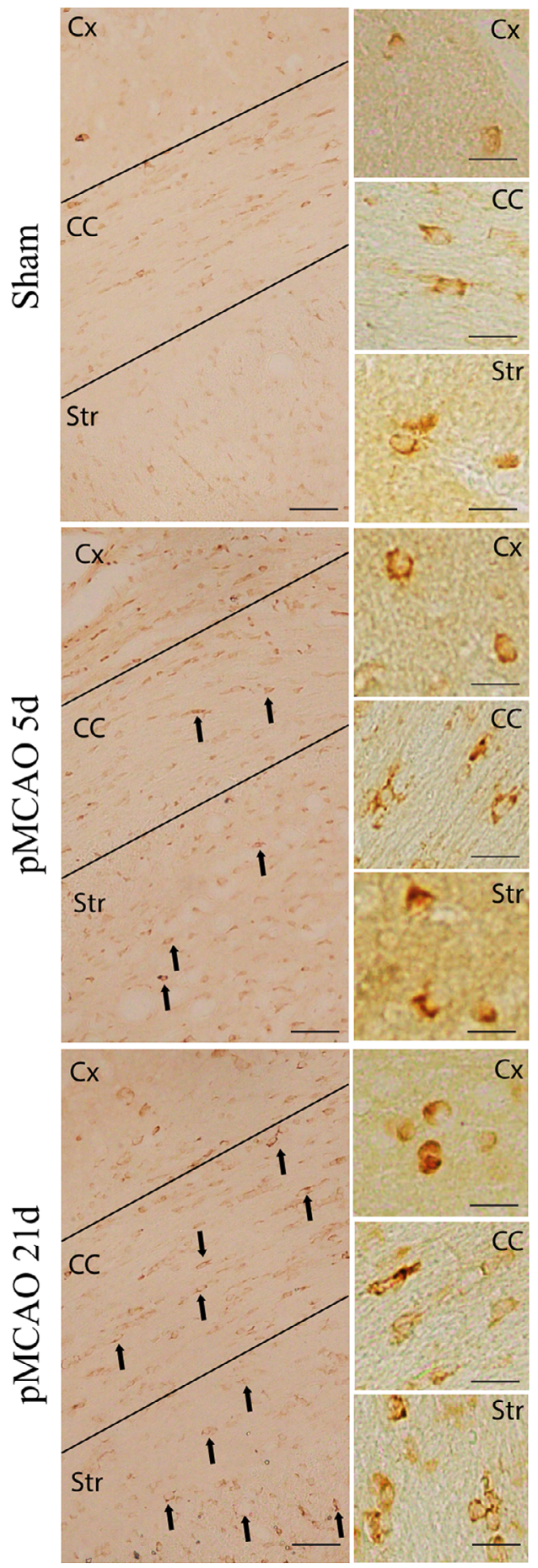

(b)
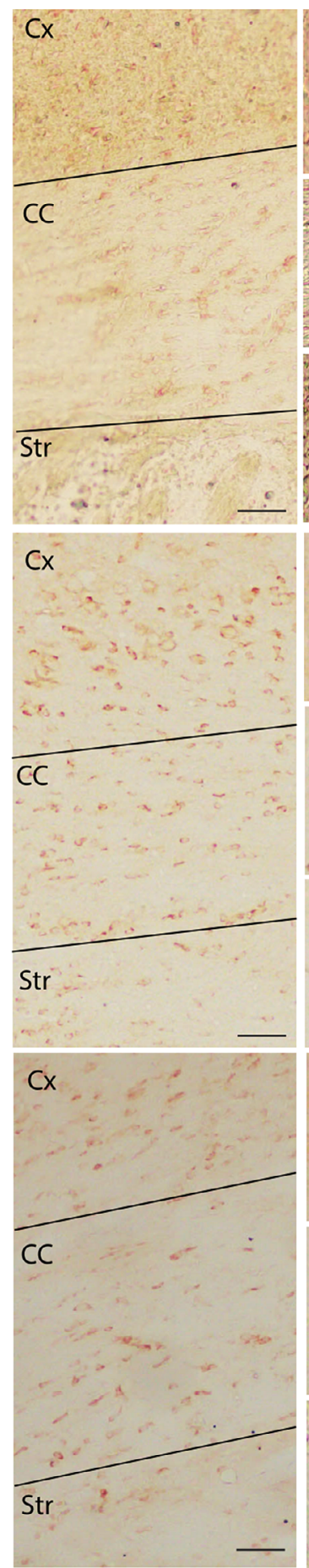

4R-Tau
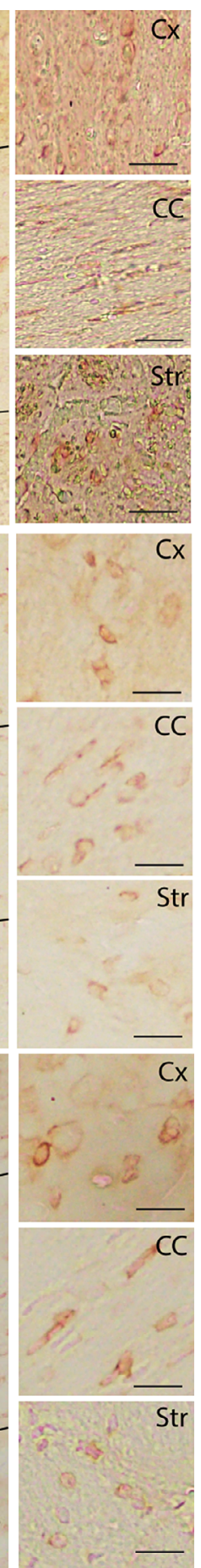

FIGURE 4 Permanent middle cerebral artery occlusion (pMCAO) induces changes in 3R-Tau levels and distribution, mainly in white matter, but not in 4R-Tau levels in the ischemic damage area.

(a) Representative coronal brain sections $(25 \mu \mathrm{m})$ corresponding to ischemic damage area (parietal region) of Sham, pMCAO-5d, and pMCAO$21 \mathrm{~d}$ rats stained with $3 \mathrm{R}-\mathrm{Tau}$ antibody, showing general view of distribution in the ipsilateral hemisphere (scale bar: $30 \mu \mathrm{m}$ ). On the right, small pictures representing a magnification of each zone (scale bar: $10 \mu \mathrm{m}$ ). (b) Immunohistochemical stained of representative coronal brain sections $(25 \mu \mathrm{m})$ of the ischemic damage area showing the general distribution of $4 \mathrm{R}-\mathrm{Tau}$ isoform of Sham, pMCAO-5d, and pMCAO-21d animals in the ipsilateral hemisphere (scale bar: $30 \mu \mathrm{m}$ ). On the right, small pictures representing a magnification of each zone (scale bar: $10 \mu \mathrm{m}$ ). Solid lines define the corpus callosum (CC). Cx, cerebral cortex; Str, striatum [Color figure can be viewed at wileyonlinelibrary.com] any significant differences in the number of Olig2 ${ }^{+}$cells between Sham and pMCAO-5d experimental groups (Figure 5a, olig2 and Figure $5 \mathrm{c}$ ). However, in the pMCAO-21d group, the number of Olig2 ${ }^{+}$ cells decreased compared with Sham rats $(274.2 \pm 12.9$ vs. $217.8 \pm 0.25 ; p=.0427$ ). The number of Olig2 ${ }^{+} / 3 R-\mathrm{Tau}^{+}$cells increased after pMCAO, being significant at 21 days postischemia (76.5 \pm 5.5 vs. $123 \pm 3 ; p=.017$ ) (Figure $5 a$,d-merged). A clear localization of $3 \mathrm{R}-\mathrm{Tau}$ in oligodendrocytic processes after pMCAO induction was also detected in both corpus callosum and cortical regions (Figures $5 \mathrm{e}$ and $6 \mathrm{e}$ ).

In the M1/M2 cortex (Figure 6a,b), the number of Olig2 ${ }^{+}$cells decreased at 21 days postischemia (115.3 \pm 1.25 vs. $62.0 \pm 9$; $p=.027$ ), probably due to an increase in the extension of the damaged area at this time (Figure 6a-Olig2 and Figure 6c). Surprisingly, the number of Olig2 ${ }^{+} / 3 \mathrm{R}-\mathrm{Tau}^{+}$cells in this region increased at 5 or 21 days postischemia $(9 \pm 1 ; 32.50 \pm 2.5 ; 18.50 \pm 1.5$; Sham vs. 


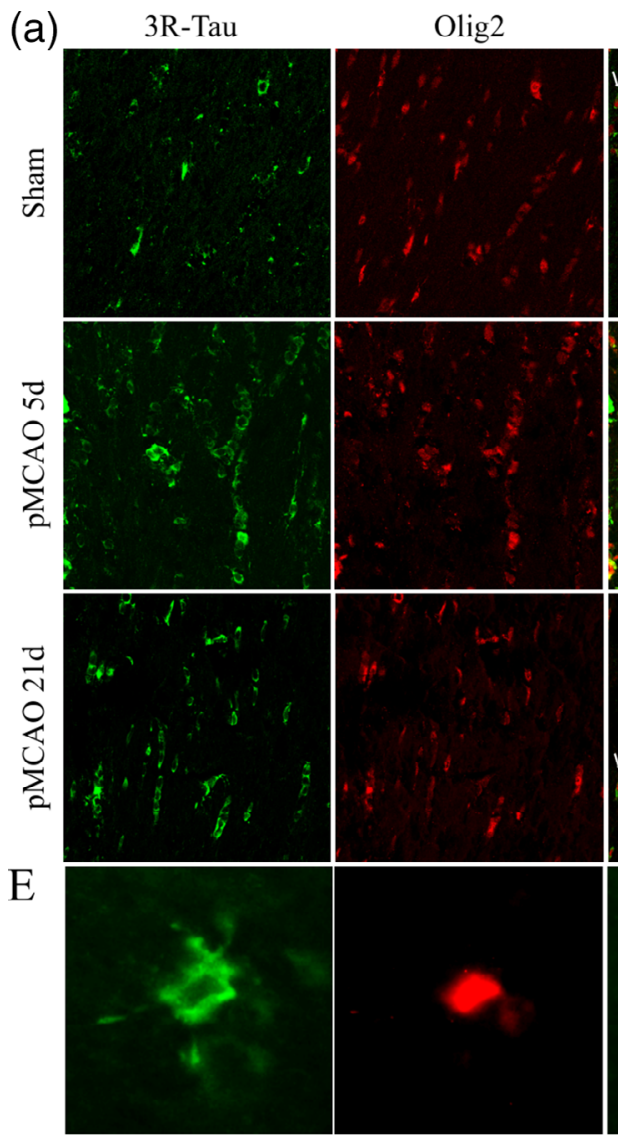

3R-Tau

Olig2

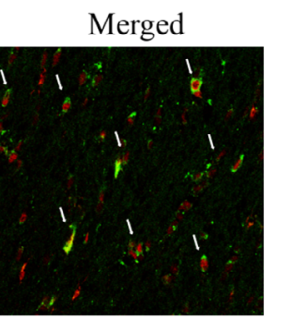

(b)

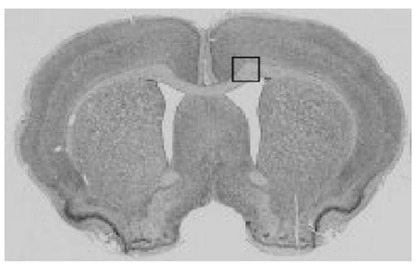

(c)

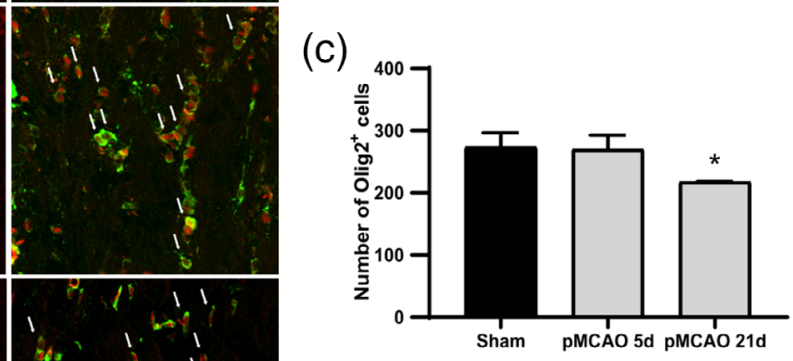

(d)

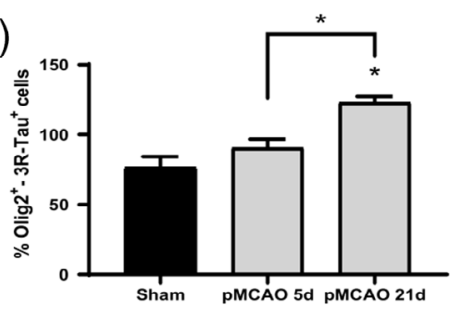

FIGURE 5 Analysis of 3R-Tau and Olig2 in the corpus callosum after ischemia. (a) Representatives confocal microphotographs corresponding to the corpus callosum of coronal brain sections $(25 \mu \mathrm{m})$ of Sham and permanent middle cerebral artery occlusion (pMCAO) rats $(5$ and 21 days after ischemia induction), stained for 3R-Tau (green, left column) and Olig2 (red, central column). Right column represents the merged channels. Scale bar: $25 \mu \mathrm{m}$. (b) Nissl-stained coronal brain section showing the selected field indicated by a rectangle. (c) Graphs represent a quantitative analysis of Olig2 ${ }^{+}$cells (total number of Olig2 ${ }^{+}$cells in the selected area). (d) Histogram represents the percentage of double positive (Olig2 ${ }^{+} / 3 \mathrm{R}-$ $\mathrm{Tau}^{+}$) respect of total Olig2 ${ }^{+}$cells. Bars represent the media \pm SEM ( $n=3-4$ rats) calculated in all images. For counting, we used four adjacent fields for each brain section and three consecutive slices from each animal. Student's $t$ test; $p$-values: $\left.{ }^{*} p<0.05\right)$. (e) Magnification of oligodendrocyte of the corpus callosum in $\mathrm{PMCAO}-5 \mathrm{~d}$ rats showing positive stain of processes

pMCAO-5d $p=.0129 ;$ Sham vs. pMCAO-21d; $p=.0342$ ) (Figure 6amerged and Figure 6d).

Our analysis of the ischemic core (S1fl: primary somatosensory cortex forelimb region; Figure 7a,b), showed an increment in the number of Olig2 ${ }^{+}$cells at 5 days postischemia compared to the Sham group (72.67 \pm 8.45 vs. $112.8 \pm 0.75 ; p=.0350$ ). Whereas the number of $3 \mathrm{R}-\mathrm{Tau}^{+}$oligodendrocytes increased in the pMCAO-5d and pMCAO-21d groups $(6.3 \pm 1.45 ; 31.33 \pm 1.45 ; 36.50 \pm 8.5$, respectively; Sham vs. pMCAO-5d $p=.0003$; Sham vs. pMCAO-21d; $p=.019)$ (Figure 7c,d).

\section{5 | The number of 3R-Tau ${ }^{+}$oligodendrocytes increases in the SVZ of the lateral ventricle after cerebral ischemia}

To determine whether the increased number of Olig2 ${ }^{+} / 3 \mathrm{R}-\mathrm{Tau}^{+}$ cells observed after pMCAO were of SVZ origin, we studied this brain region (Figure 8a,b) using sections from Sham, pMCAO-5d, and pMCAO-21d animals. We found that the number of Olig2 ${ }^{+}$ cells increased 5 days postischemia $(38.75 \pm 2.59 ; p=.0076)$ and remains the same as Sham at 21 days pMCAO (Figure 8 e). Similar to our previous findings in the corpus callosum and cerebral cortex, the number of $3 \mathrm{R}-\mathrm{Tau}^{+}$oligodendrocytes increased significantly after PMCAO $(3.33 \pm 0.33 ; \quad 6.66 \pm 0.3 ; \quad 17.5 \pm 1.5$, respectively; Sham vs. pMCAO-5d $p=.029$; Sham vs. $\mathrm{pMCAO}-21 \mathrm{~d}$; $p<.0001$ ) (Figure 8a,f). To examine whether oligodendrogenesis was induced by pMCAO, we injected rats with a dose of IdU $6 \mathrm{hr}$ postsurgery and evaluated the ischemic brain at 5 days postischemia. The IdU stain of both the SVZ and corpus callosum increased, thereby indicating that cell division was induced (Figure 8c). Double staining using anti-IdU and anti-Olig2 antibodies revealed cells with double staining, thereby confirming pMCAO-induced oligodendrogenesis in the SVZ (Figure 8d). These results suggest that some oligodendrocytes have an SVZ origin after pMCAO. 
(a)
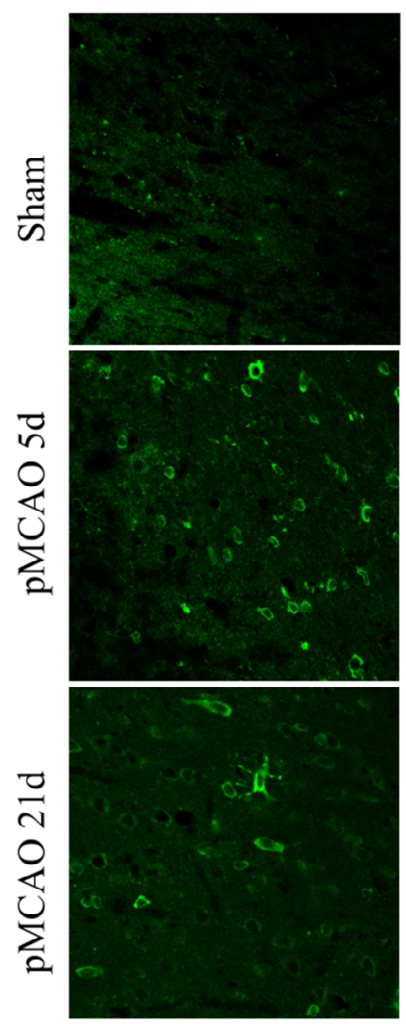

(e)

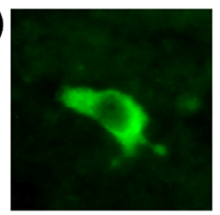

3R-Tau

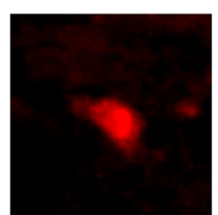

Olig2
Olig2
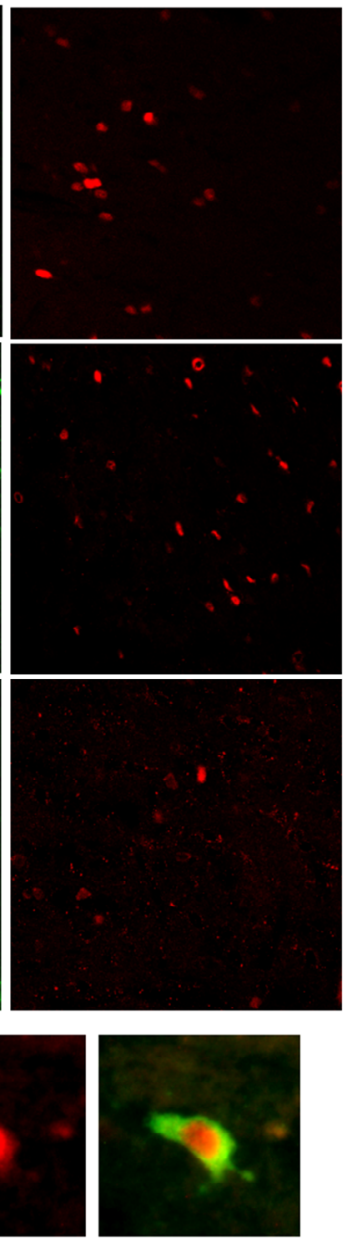

Merged
Merged

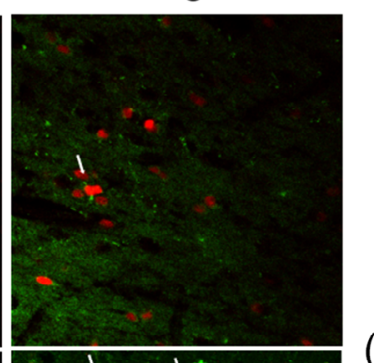

(b)

(c)
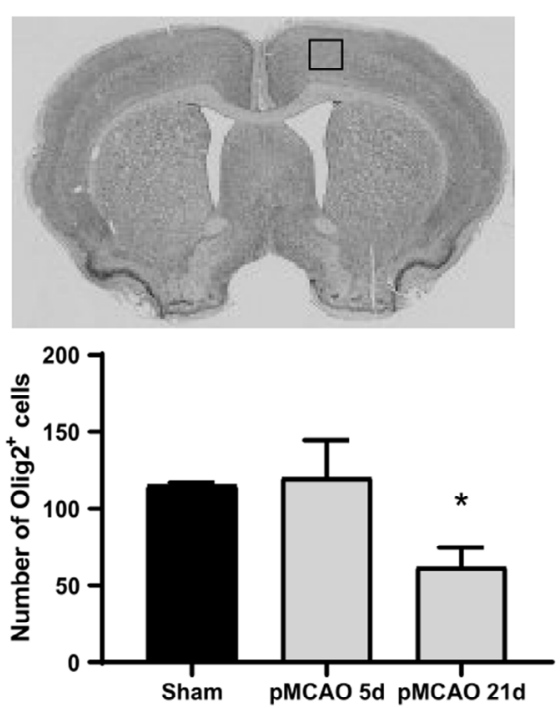

(d)

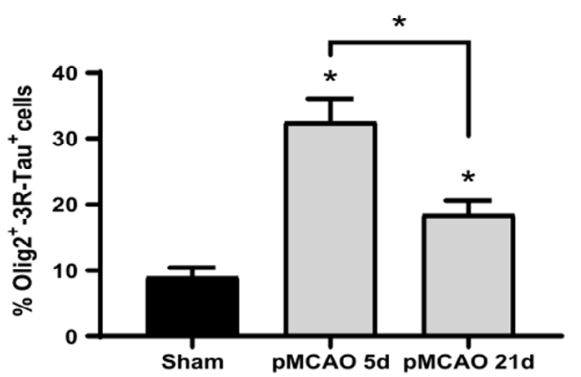

FIGURE 6 Analysis of 3R-Tau and Olig2 distribution in the cerebral cortex (penumbra region) after permanent middle cerebral artery occlusion (pMCAO). (a) Representatives confocal microphotographs corresponding to cerebral cortex (M1/M2 region) of coronal brain sections $(25 \mu \mathrm{m})$ of Sham and pMCAO rats (5 and 21 days after ischemia induction), stained for 3R-Tau (green, left column) and Olig2 (red, central column). Right column represents the merged channels. Scale bar: $25 \mu \mathrm{m}$. (b) Nissl-stained coronal brain section showing the selected field indicated by a solid rectangle. (c) Graphs represent a quantitative analysis of Olig2 ${ }^{+}$cells (total number of Olig2 ${ }^{+}$cells in the selected area). (d) Histogram represents the percentage of double positive (Olig2 ${ }^{+} / 3 \mathrm{R}-\mathrm{Tau}^{+}$) respect of total Olig2 ${ }^{+}$cells. Bars represent the media \pm SEM ( $n=3-4$ rats) calculated in all images. For counting, we used four adjacent fields for each brain section and three consecutive slices from each animal. Student's $t$ test; $p$-values: ${ }^{*} p<0.05$. (e) Magnification of cortical oligodendrocyte in pMCAO-21d rats showing positive stain of processes

\section{DISCUSSION}

The impact of ischemic damage after stroke has been widely studied in neurons (gray matter), but little is known about its impact on white matter. Data in human and experimental stroke models reveal that the adult brain shows self-repair capacity, which is reflected in a spontaneous improvement of neurological state, motor or sensorial capabilities after the insult (Dewar et al., 2003; Ligon, Fancy, Franklin, \& Rowitch, 2006; Zhang et al., 2013). Little attention has been paid to this brain self-repair process. However, a greater understanding of this mechanism could provide a new therapeutic approach through which to enhance endogenous brain repair mechanisms.

Our results indicated that the damaged area (ischemic core) increased in size over time, occupying almost half of the hemisphere affected at 21 days post-pMCAO. In parallel, the neurological score of ischemic rats improved spontaneously, almost reaching Sham values at 21 days postischemia. These findings indicate that the CNS responds to pMCAO by triggering a self-repair mechanism, which serves to maintain brain function, even when the tissue is damaged.

A number of mechanisms involved in brain repair during recovery have been proposed, namely neurogenesis, angiogenesis, reactive gliosis, and oligodendrogenesis (Perez-Alvarez \& Wandosell, 2016; Zhang \& Chopp, 2009). The role of neurogenesis and angiogenesis after cerebral ischemia have been studied widely (Caglayan et al., 2019; Peng et al., 2019; Perez-Alvarez \& Wandosell, 2016), pointing out that both mechanisms were induced after ischemia and improves functional recovery and tissue remodeling. Furthermore, potentiation of these endogenous repair mechanisms after ischemic 
(a)
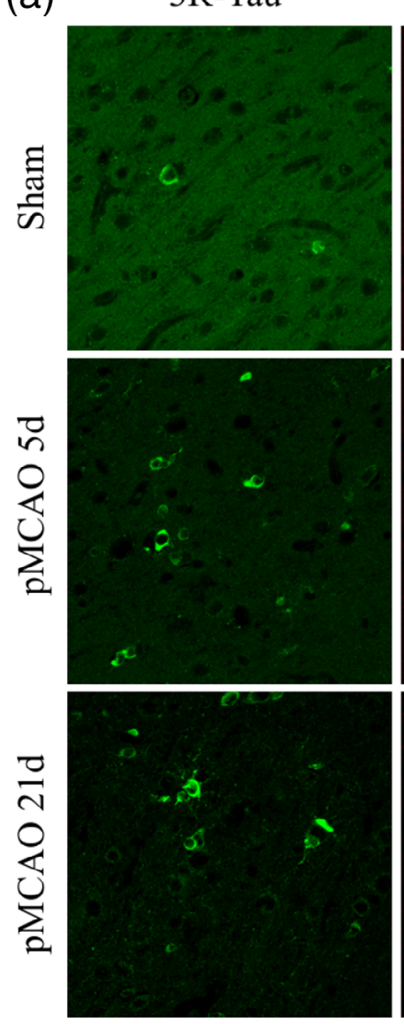

Olig2
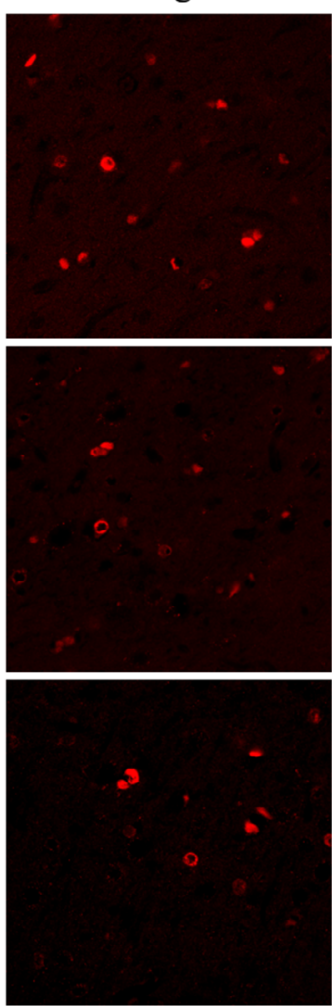

Merged
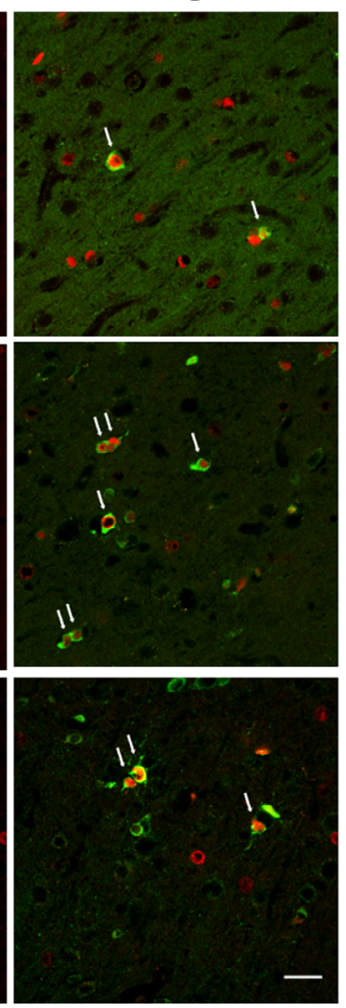

(b)

(c)
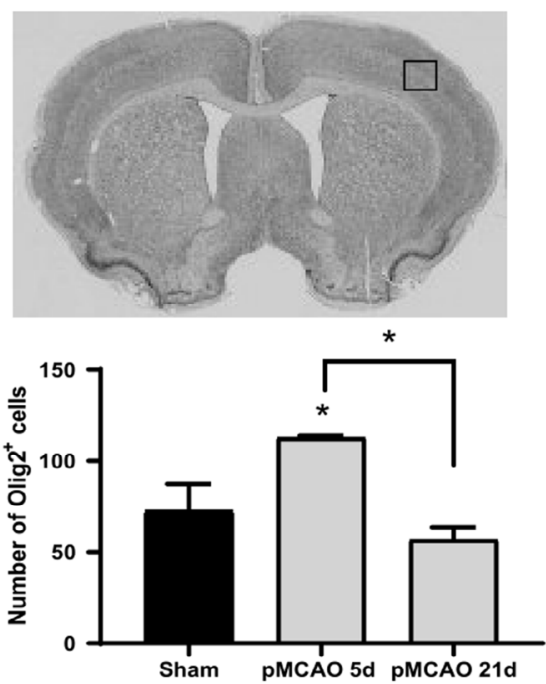

(d)

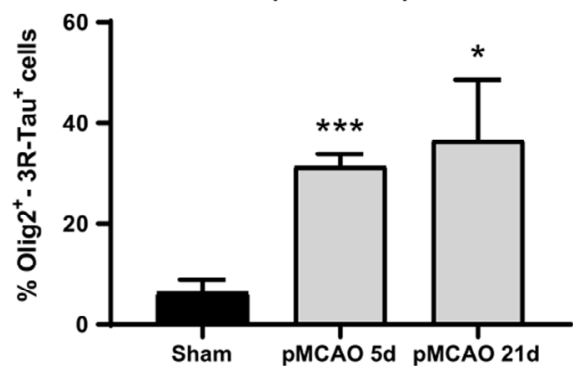

FIGURE 7 Analysis of 3R-Tau and Olig2 distribution in the ischemic core after permanent middle cerebral artery occlusion (pMCAO). (a) Representative confocal microphotographs corresponding to the cerebral cortex (S1fl: primary somatosensory cortex forelimb region) of coronal brain sections $(25 \mu \mathrm{m})$ of Sham and pMCAO rats ( 5 and 21 days after ischemia induction), stained for 3R-Tau (green, left column) and Olig2 (red, central column). Right column represents the merged channels. Scale bar: $25 \mu \mathrm{m}$. (b) Nissl-stained coronal brain section showing the selected field indicated by a rectangle. (c) Graphs represent a quantitative analysis of Olig2 ${ }^{+}$cells (total number of Olig2 ${ }^{+}$cells in the selected area). (d) Histogram represents the percentage of double positive (Olig2 ${ }^{+} / 3 \mathrm{R}-\mathrm{Tau}^{+}$) respect of total Olig2 ${ }^{+}$cells. Bars represent the media $\pm S E M$ ( $n=3-4$ rats) calculated in all images. For counting, we used four adjacent fields for each brain section and three consecutive slices from each animal. Student's $t$ test; $p$-values: $\left.{ }^{*} p<0.05 ;{ }^{* * *} p<0.01\right)$

injury ameliorates damage. The relationship between reactive gliosis and recovery after ischemia is a subject under debate (Perez-Alvarez \& Wandosell, 2016; Qin et al., 2019). However, few studies have addressed oligodendrogenesis and the underlying mechanisms or the exact role of this process after stroke (Dewar et al., 2003; Jiang et al., 2011; Liu et al., 2012; LoPresti et al., 1995; Mandai et al., 1997; Zhang et al., 2013). As the only myelin-forming cells in the CNS, oligodendrocytes are highly vulnerable to ischemia (Dewar et al., 2003; Domercq et al., 2010; Ligon et al., 2006; Matute et al., 2006; Pantoni et al., 1996) and any damage to them impairs myelin production and correct axonal function, thereby worsening neuronal damage caused by ischemia. Some studies have described that the preservation of white matter integrity reduces ischemic damage after stroke and improves recovery (Dai et al., 2019; Liu et al., 2012; Zhao et al., 2019). Also, it has been suggested that oligodendrocyte precursor cells and oligodendrocytes have trophic functions after this insult (Bonfanti et al., 2017; Miyamoto et al., 2014).

Oligodendrocytes have a complex cytoskeleton containing a network of microtubules and microfilaments. During their maturation, these cells undergo profound morphological changes that involve processes that require cytoskeleton reorganization and a dynamic microtubule network, both of which are regulated by MAPs, including tau (Gorath et al., 2001; Ness, Valentino, Mclver, \& Goldberg, 2005). Tau plays a key role in microtubule stabilization by binding through its three or four repetitive MBDs (LoPresti, 2018). The balance between tau isoforms enables the cytoskeleton to respond to changes in neuronal functions. Previous studies suggest that the 3R-Tau isoform results in less stable microtubule binding and a more dynamic cytoskeleton (Wang \& Mandelkow, 2015). In contrast, 4R-Tau shows higher affinity and more efficiency at promoting microtubule assembly. 3R-Tau is abundant in the neonatal brain and in immature oligodendrocytes cultured in vitro, whereas $4 \mathrm{R}-\mathrm{Tau}$ is profuse in the adult brain and mature oligodendrocyte cultures (Gorath et al., 2001; LoPresti, 2018). Our in vivo results, using immunofluorescence with isoform-specific antibodies, indicate that 3R-Tau and 4R-Tau are present in the brains of healthy adult rats and that they have a similar distribution in interfascicular oligodendrocytes (mature) of the corpus callosum, mainly in the soma around the nucleus, a similar pattern of 

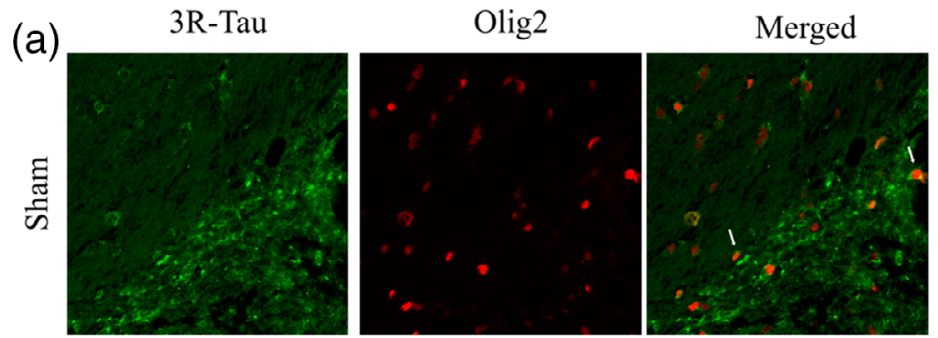

(b)
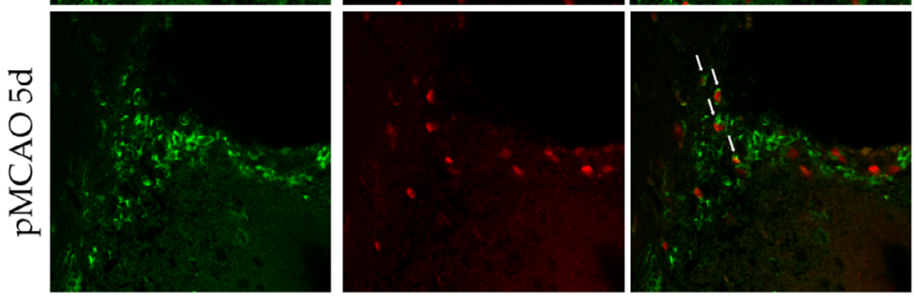

(c)

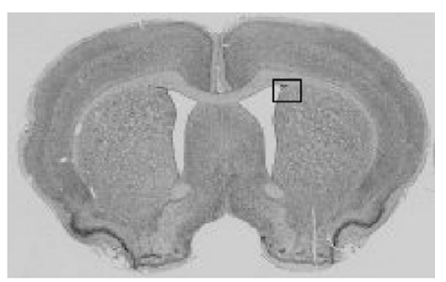

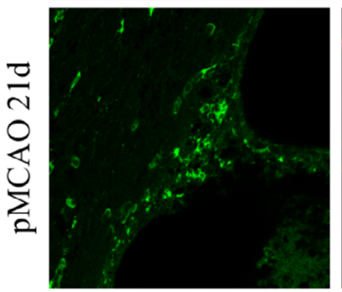

(d)

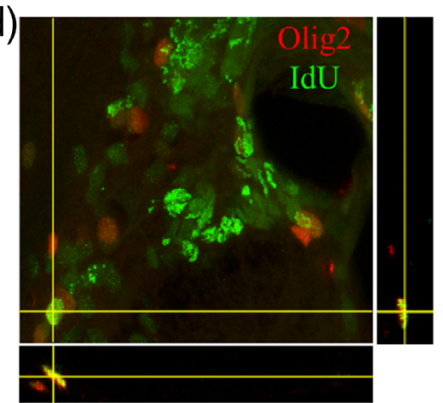

pMCAO 5d

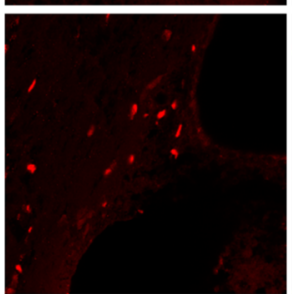

(e)
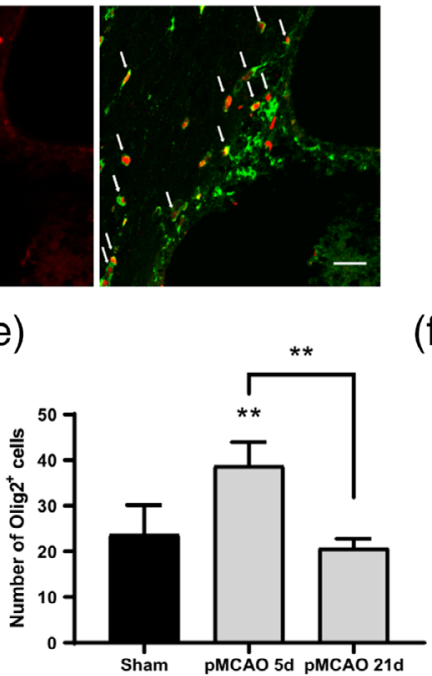

Sham

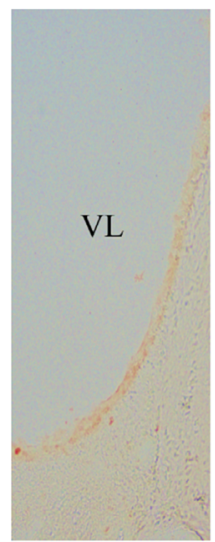

pMCAO 5d

(f)

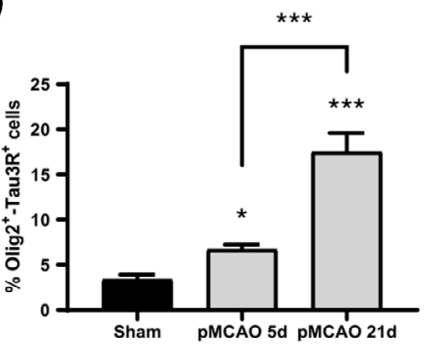

FIGURE 8 The number of 3R-Tau ${ }^{+}$oligodendrocytes increases in the subventricular zone (SVZ) of the lateral ventricle (VL) after permanent middle cerebral artery occlusion (pMCAO). (a) Representative confocal microphotographs corresponding to the SVZ of the VL of Sham and pMCAO rats ( 5 and 21 days after ischemia induction), stained for 3R-Tau (green, left column) and Olig2 (red, central column). Right column represents the merged channels. Scale bar: $25 \mu \mathrm{m}$. (b) Nissl-stained coronal brain section showing the selected field indicated by a rectangle. (c) Representative microscope images of coronal brain sections $(25 \mu \mathrm{m})$ of the VL of Sham and pMCAO-5d animals stained with anti-IdU antibody. Arrows: point to positive cells in the SVZ zone and some in ventral corpus callosum close to SVZ. (d) Orthogonal view of double stained cell (Olig2/ldU) in the SVZ of the VL of pMCAO-5d rats. (e) Graphs represent a quantitative analysis of Olig2 ${ }^{+}$cells (total number of Olig2 ${ }^{+}$cells in the selected area). (f) Histogram represents the percentage of double positive (Olig2 ${ }^{+} / 3 \mathrm{R}-\mathrm{Tau}^{+}$) respect of total Olig2 ${ }^{+}$cells. For counting, we used two different but adjacent fields for each brain section and three consecutive slices of each animal. (Student's $t$ test; $p$-values: ${ }^{*} p<0.05$; ${ }^{* *} p<0.01 ;{ }^{* * *} p<0.001$ )

distribution to that found in mouse brain oligodendrocytes using antitotal tau antibodies (Kubo et al., 2019). In addition, 3R-Tau antibody clearly stained the tips of processes of some oligodendrocytes in white matter. These results demonstrate that mature brain oligodendrocytes in the white matter contain both 3R- and 4R-Tau isoforms and that these show a similar pattern of distribution. In contrast, in vitro data (Gorath et al., 2001) using RT-PCR and in situ hybridization reveals a greater amount of 4R-Tau mRNA compared to 3R-Tau mRNA in mature oligodendrocytes. This apparent discrepancy highlights the importance of combining methodological approaches to analyze the presence and distribution of proteins versus mRNA. Our results show that the distribution of $4 \mathrm{R}-\mathrm{Tau}$ is broader and mainly neuronal in gray matter, whereas $3 \mathrm{R}-\mathrm{Tau}$ is predominantly present in oligodendrocytes localized in the soma and tips of processes. Here we have shown that pMCAO has a different impact on 3R-Tau and 4RTau levels at 5 and 21 days postinjury. Western blot and histological techniques revealed a notable increase in 3R-Tau protein at 5 and 21 days post-pMCAO, whereas 4R-Tau levels did not change significantly in white or in gray matter with respect to Sham animals. In good agreement with these data, the 3R/4R mRNA ratio, in samples containing cerebral cortex and the underlying corpus callosum, increased at 2 days post-pMCAO, indicating that ischemia induces an 
imbalance in these isoforms, due in part to alterations in the splicing of tau Exon 10, thereby resulting in the induction of 3R-Tau. Similar results in neurons, using a transient-MCAO (MCAO) model and short times postischemia, have been reported (Suh et al., 2010). Some previous studies described an increment in total tau immunoreactivity in oligodendrocytes after short times (2, 6, $24 \mathrm{hr}$ ) of pMCAO induction (Dewar \& Dawson, 1995; Irving et al., 2001; Irving, Yatsushiro, McCulloch, \& Dewar, 1997). Altogether, our results demonstrate that cerebral ischemia affects the two tau isoforms in a different manner and that this imbalance is maintained for a long time (until 21 days). Our findings also reveal that the rise in tau levels reported previously is due to an increase in 3R-Tau and not 4R-Tau, as ischemia alters the splicing of tau Exon 10 in oligodendrocytes, similar to what has been described in neurons (Suh et al., 2010).

We also found that the cellular distribution of 3R-Tau is modified after 5 and 21 days of pMCAO, changing from mainly cytoplasmic staining, around the nucleus, to being present also at the tips of the processes. Our results suggest that pMCAO increases 3R-Tau expression, thereby inducing changes in microtubule dynamics, which probably allow oligodendrocytes to change the orientation of their processes or to migrate to damaged areas in order to myelinateinjured axons. The presence of 3R-Tau at the tips of the processes suggests an increase in oligodendrocytes with "axon-free" extensions, which could have the potential to myelinate-injured axons. In this regard, we propose 3R-Tau as a good marker of premyelinating oligodendrocytes (Zhang et al., 2013).

Stroke induces mature oligodendrocyte damage associated with loss of axons at initial stages of injury (Pantoni et al., 1996; Zhang et al., 2013). However, at later stages, there is an increase in oligodendrocyte progenitor cells, which allows some of them to myelinate in the peri-infarct region (Jiang et al., 2011; LoPresti, 2018; Mandai et al., 1997; Zhang et al., 2012). Those studies were carried out using mouse or rats with $\mathrm{tMCAO}$ or pMCAO induction and the ischemic effects were examined from hours to 5 days after damage. In the present article, we extended the study time to 21 days. Our results show that after 5 days of pMCAO the number of oligodendrocytes in the corpus callosum was similar to that present in the Sham group. This population tended to increase in the penumbra (M1/M2 area) and significantly increased in the ischemic core (S1fl zone) and SVZ. However, after 21 days of pMCAO, the number of Olig- $2^{+}$cells decreases comparing with Sham group in all studied areas. These results at 5 days postinjury are in agreement with those obtained by (Mandai et al., 1997) using in situ hybridization with mRNA-PLP or mRNAMBP. Our results obtained at 21 days post-pMCAO demonstrate that long-lasting ischemia reduces the number of oligodendrocytes, probably because lengthy exposure to oxygen/glucose deprivation severely affects these cells. These findings suggest that ischemic damage to oligodendrocytes after pMCAO occurs after neuronal injury, as described before (Gresle, Jarrott, Jones, \& Callaway, 2006) and suggest that a delayed therapeutic intervention may have benefit for the protection of oligodendrocytes.

Nevertheless, in our pMCAO model, the number of 3R-Tau ${ }^{+}$oligodendrocytes increased at 5 and 21 days postinjury in all the brain regions analyzed. Almost $25 \%$ of the oligodendrocytes found in gray matter were 3R-Tau ${ }^{+}$after ischemia. The increase in 3R-Tauexpressing oligodendrocytes was also observed in the SVZ, being more evident in the pMCAO-21d group. Using IdU/Olig2 antibodies for double staining, we have demonstrated that some oligodendrocytes were generated $6 \mathrm{hr}$ after cerebral ischemia. Thus, we can conclude that after $6 \mathrm{hr}$ of pMCAO, cell division occurs in the SVZ zone and that some of these cells differentiate into oligodendrocytes. This would explain, in part, the increase in oligodendrocytes that we detected in the damaged area. The observation of positive staining for the oligodendrocytes in the SVZ of the pMCAO-5d and pMCAO-21d animals leads us to propose that the presence of this tau isoform generates a more dynamic cytoskeleton that allows oligodendrocyte migration to the ischemic tissue. However, we cannot rule out the possibility that the $3 \mathrm{R}-\mathrm{Tau}^{+}$oligodendrocytes found in gray matter after pMCAO derive from a "reserve pool" of progenitors $\left(\mathrm{NG}^{+}\right)$ maintained in adult brain for the purpose of repairing processes, as other authors have suggested (Bonfanti et al., 2017; Bradl \& Lassmann, 2010; Dai et al., 2019).

We propose that cerebral ischemia triggers an endogenous mechanism, involving 3R-Tau, that could induce oligodendrocytes to myelinate-injured axons. This event occurs in parallel to an improvement in the neurological score of pMCAO rats. These cellular mechanisms seem to be based, in part, on alterations in tau isoform expression, which modulate the dynamics of the cytoskeleton and induce changes in cellular morphology. The molecular mechanisms that underlie this phenomenon could provide the basis for designing new therapeutic strategies focused on enhancing endogenous mechanisms of brain repair based on glia cells and not only on neurons. Bearing in mind that therapeutic approaches to alleviate the devastating effects of cerebral ischemia used so far in animal models are based only in neurons, and that none of them have been approved the clinical trials, discover alternative mechanisms of neuroprotection based on glial cells emerge as an exciting alternative.

\section{ACKNOWLEDGMENTS}

This study was funded by grants from Spanish Ministry of Economy and Competitiveness (BFU-2008-03980 and BFU2016-77885-P), Comunidad de Madrid (S2017/BMD-3700), Centro de Investigación Biomédica en Red sobre Enfermedades Neurodegenerativas (CIBERNED, ISCIII), and an institutional grant from the Fundación R. Areces. The authors are grateful to all the members of the Lab at the Centro de Biología Molecular "Severo Ochoa" (CBM SO) and Dpto de Biología (Fisiología Animal) for thoughtful discussions during the preparation of this manuscript. The authors thank the Confocal Microscopy Service at the CBMSO for technical assistance and guidance.

\section{CONFLICT OF INTEREST}

The authors declare that there is no conflict of interest that could be perceived as detrimental to the impartiality of the research reported. 


\section{DATA AVAILABILITY STATEMENT}

The data that support the findings of this study are available from the corresponding author upon reasonable request.

\section{ORCID}

María José Pérez-Alvarez (D) https://orcid.org/0000-0001-8334-8085

\section{REFERENCES}

Andreadis, A., Brown, W. M., \& Kosik, K. S. (1992). Structure and novel exons of the human tau gene. Biochemistry, 31(43), 10626-10633. https://doi.org/10.1021/bi00158a027

Avila, J., Lucas, J. J., Pérez, M., \& Hernandez, F. (2004). Role of tau protein in both physiological and pathological conditions. Physiological Reviews, 84(2), 361-384. https://doi.org/10.1152/physrev.00024.2003

Baumann, N., \& Pham-Dinh, D. (2001). Biology of oligodendrocyte and myelin in the mammalian central nervous system. Physiological Reviews, 81(2), 871-927. https://doi.org/10.1152/physrev.2001.81.2.871

Benjamin, E. J., Muntner, P., Alonso, A., Bittencourt, M. S., Callaway, C. W., Carson, A. P., ... Virani, S. S. (2019). Heart disease and stroke statistics-2019 update: A report from the American Heart Association. Circulation, 139(10), e56-e528. https://doi.org/10.1161/ CIR.0000000000000659

Bonfanti, E., Gelosa, P., Fumagalli, M., Dimou, L., Viganò, F., Tremoli, E., ... Abbracchio, M. P. (2017). The role of oligodendrocyte precursor cells expressing the GPR17 receptor in brain remodeling after stroke. Cell Death and Disease, 8, e2871. https://doi.org/10.1038/cddis.2017.256

Bradl, M., \& Lassmann, H. (2010). Oligodendrocytes: Biology and pathology. Acta Neuropathologica, 119(1), 37-53. https://doi.org/10.1007/ s00401-009-0601-5

Butt, A. M., \& Dinsdale, J. (2005). Fibroblast growth factor 2 mediated disruption of myelin-forming oligodendrocytes in vivo is associated with increased tau immunoreactivity. Neuroscience Letters, 375(1), 28-32. https://doi.org/10.1016/j.neulet.2004.10.060

Caglayan, A. B., Beker, M. C., Caglayan, B., Yalcin, E., Caglayan, A., Yulug, B., ... Kilic, E. (2019). Acute and post-acute neuromodulation induces stroke recovery by promoting survival signaling, neurogenesis, and pyramidal tract plasticity. Frontiers in Cellular Neuroscience, 13 (144). https://doi.org/10.3389/fncel.2019.00144

Dai, X., Chen, J., Xu, F., Zhao, J., Cai, W., Sun, Z., ... Hu, X. (2019). TGF $\alpha$ preserves oligodendrocyte lineage cells and improves white matter integrity after cerebral ischemia. Journal of Cerebral Blood Flow and Metabolism, O(0), 0271678X19830791. https://doi.org/10.1177/ 0271678x19830791

del Puerto, A., Fronzaroli-Molinieres, L., Perez-Alvarez, M. J., Giraud, P., Carlier, E., Wandosell, F., ... Garrido, J. J. (2014). ATP-P2X7 receptor modulates axon initial segment composition and function in physiological conditions and brain injury. Cerebral Cortex, 25(8), 2282-2294. https://doi.org/10.1093/cercor/bhu035

Dewar, D., \& Dawson, D. (1995). Tau protein is altered by focal cerebral ischaemia in the rat: An immunohistochemical and immunoblotting study. Brain Research, 684(1), 70-78. https://doi.org/10.1016/00068993(95)00417-O

Dewar, D., Underhill, S. M., \& Goldberg, M. P. (2003). Oligodendrocytes and ischemic brain injury. Journal of Cerebral Blood Flow \& Metabolism, 23(3), 263-274. https://doi.org/10.1097/01.Wcb.0000053472. 41007.F9

Domercq, M., Perez-Samartin, A., Aparicio, D., Alberdi, E., Pampliega, O., \& Matute, C. (2010). P2X7 receptors mediate ischemic damage to oligodendrocytes. Glia, 58(6), 730-740. https://doi.org/10.1002/glia. 20958

Goedert, M., \& Jakes, R. (1990). Expression of separate isoforms of human tau protein: Correlation with the tau pattern in brain and effects on tubulin polymerization. The EMBO Journal, 9(13), 4225-4230.
Gorath, M., Stahnke, T., Mronga, T., Goldbaum, O., \& RichterLandsberg, C. (2001). Developmental changes of tau protein and mRNA in cultured rat brain oligodendrocytes. Glia, 36(1), 89-101. https://doi.org/10.1002/glia.1098

Gresle, M. M., Jarrott, B., Jones, N. M., \& Callaway, J. K. (2006). Injury to axons and oligodendrocytes following endothelin-1-induced middle cerebral artery occlusion in conscious rats. Brain Research, 1110(1), 13-22. https://doi.org/10.1016/j.brainres.2006.06.111

Irving, E., Bentley, D., \& Parsons, A. (2001). Assessment of white matter injury following prolonged focal cerebral ischaemia in the rat. Acta Neuropathologica, 102(6), 627-635. https://doi.org/10.1007/ s004010100416

Irving, E. A., Yatsushiro, K., McCulloch, J., \& Dewar, D. (1997). Rapid alteration of tau in oligodendrocytes after focal ischemic injury in the rat: Involvement of free radicals. Journal of Cerebral Blood Flow and Metabolism, 17(6), 612-622. https://doi.org/10.1097/00004647199706000-00003

Jiang, L., Shen, F., Degos, V., Schonemann, M., Pleasure, S. J., Mellon, S. H., ... Su, H. (2011). Oligogenesis and oligodendrocyte progenitor maturation vary in different brain regions and partially correlate with local angiogenesis after ischemic stroke. Translational Stroke Research, 2(3), 366-375. https://doi.org/10.1007/s12975-011-0078-0

Kubo, A., Misonou, H., Matsuyama, M., Nomori, A., Wada-Kakuda, S., Takashima, A., ... Miyasaka, T. (2019). Distribution of endogenous normal tau in the mouse brain. Journal of Comparative Neurology, 527(5), 985-998. https://doi.org/10.1002/cne.24577

Ligon, K. L., Fancy, S. P. J., Franklin, R. J. M., \& Rowitch, D. H. (2006). Olig gene function in CNS development and disease. Glia, 54(1), 1-10. https://doi.org/10.1002/glia.20273

Liu, C.-W. A., Lee, G., \& Jay, D. G. (1999). Tau is required for neurite outgrowth and growth cone motility of chick sensory neurons. Cell Motility, 43(3), 232-242. https://doi.org/10.1002/(sici)1097-0169(1999) 43:3<232::aid-cm6>3.0.co;2-7

Liu, X. S., Chopp, M., Kassis, H., Jia, L. F., Hozeska-Solgot, A., Zhang, R. L., ... Zhang, Z. G. (2012). Valproic acid increases white matter repair and neurogenesis after stroke. Neuroscience, 220, 313-321. https://doi. org/10.1016/j.neuroscience.2012.06.012

LoPresti, P. (2018). Tau in oligodendrocytes takes neurons in sickness and in health. International Journal of Molecular Sciences, 19(8), 2408.

LoPresti, P., Szuchet, S., Papasozomenos, S. C., Zinkowski, R. P., \& Binder, L. I. (1995). Functional implications for the microtubuleassociated protein tau: Localization in oligodendrocytes. Proceedings of the National Academy of Sciences, 92(22), 10369-10373. https://doi. org/10.1073/pnas.92.22.10369

Mandai, K., Matsumoto, M., Kitagawa, K., Matsushita, K., Ohtsuki, T., Mabuchi, T., ... Yanagihara, T. (1997). Ischemic damage and subsequent proliferation of oligodendrocytes in focal cerebral ischemia. Neuroscience, 77(3), 849-861. https://doi.org/10.1016/S0306-4522 (96)00517-9

Mateos, L., Perez-Alvarez, M. J., \& Wandosell, F. (2016). Angiotensin II type-2 receptor stimulation induces neuronal VEGF synthesis after cerebral ischemia. Biochimica et Biophysica Acta (BBA) - Molecular Basis of Disease, 1862(7), 1297-1308. https://doi.org/10.1016/j.bbadis. 2016.03.013

Matute, C., Domercq, M., \& Sánchez-Gómez, M.-V. (2006). Glutamatemediated glial injury: Mechanisms and clinical importance. Glia, 53(2), 212-224. https://doi.org/10.1002/glia.20275

Merkley, C. M., Jian, C., Mosa, A., Tan, Y.-F., \& Wojtowicz, J. M. (2014). Homeostatic regulation of adult hippocampal neurogenesis in aging rats: Long-term effects of early exercise. Frontiers in Neuroscience, 8 (174). https://doi.org/10.3389/fnins.2014.00174

Migheli, A., Butler, M., Brown, K., \& Shelanski, M. (1988). Light and electron microscope localization of the microtubule- associated tau protein in rat brain. The Journal of Neuroscience, 8(6), 1846-1851. https:// doi.org/10.1523/jneurosci.08-06-01846.1988 
Mitsios, N., Gaffney, J., Kumar, P., Krupinski, J., Kumar, S., \& Slevin, M. (2006). Pathophysiology of acute Ischaemic stroke: An analysis of common signalling mechanisms and identification of new molecular targets. Pathobiology, 73(4), 159-175. https://doi.org/10.1159/ 000096017

Miyamoto, N., Pham, L.-D. D., Seo, J. H., Kim, K.-W., Lo, E. H., \& Arai, K. (2014). Crosstalk between cerebral endothelium and oligodendrocyte. Cellular and Molecular Life Sciences, 71(6), 1055-1066. https://doi.org/ 10.1007/s00018-013-1488-9

Müller, R., Heinrich, M., Heck, S., Blohm, D., \& Richter-Landsberg, C. (1997). Expression of microtubule-associated proteins MAP2 and tau in cultured rat brain oligodendrocytes. Cell and Tissue Research, 288(2), 239-249. https://doi.org/10.1007/s004410050809

Ness, J. K., Valentino, M., Mclver, S. R., \& Goldberg, M. P. (2005). Identification of oligodendrocytes in experimental disease models. Glia, 50(4), 321-328. https://doi.org/10.1002/glia.20206

Pantoni, L., Garcia, J. H., \& Gutierrez, J. A. (1996). Cerebral white matter is highly vulnerable to ischemia. Stroke, 27(9), 1641-1647. https://doi. org/10.1161/01.STR.27.9.1641

Paxinos, G., \& Watson, C. (2007). The rat brain in stereotaxic coordinates (6th ed.). San Diego, CA: Academic Press.

Peng, L., Yin, J., Ge, M., Wang, S., Xie, L., Li, Y., ... Ma, K. (2019). Isoflurane post-conditioning ameliorates cerebral ischemia/reperfusion injury by enhancing angiogenesis through activating the Shh/Gli signaling pathway in rats. Frontiers in Neuroscience, 13(321). https://doi.org/10. 3389/fnins.2019.00321

Perez-Alvarez, M. J., Mateos, L., Alonso, A., \& Wandosell, F. (2015). Estradiol and progesterone administration after pMCAO stimulates the neurological recovery and reduces the detrimental effect of ischemia mainly in hippocampus. Molecular Neurobiology, 52(3), 1690-1703. https://doi.org/10.1007/s12035-014-8963-7

Perez-Alvarez, M. J., \& Wandosell, F. (2016). Stroke and neuroinflammation: Role of sexual hormones. Current Pharmaceutical Design, 22(10), 1334-1349. https://doi.org/10.2174/138161282210160304112834

Qin, C., Zhou, L.-Q., Ma, X.-T., Hu, Z.-W., Yang, S., Chen, M., ... Tian, D.-S. (2019). Dual functions of microglia in ischemic stroke. Neuroscience Bulletin., 35, 921-933. https://doi.org/10.1007/s12264-019-00388-3

Richter-Landsberg, C. (2016). Protein aggregate formation in oligodendrocytes: tau and the cytoskeleton at the intersection of neuroprotection and neurodegeneration. Biological Chemistry, 397, 185.

Schindelin, J., Arganda-Carreras, I., Frise, E., Kaynig, V., Longair, M., Pietzsch, T., ... Cardona, A. (2012). Fiji: An open-source platform for biological-image analysis. Nature Methods, 9, 676-682. https://doi. org/10.1038/nmeth.2019

Suh, J., Im, D. S., Moon, G. J., Ryu, K. S., de Silva, R., Choi, I. S., ... Gwag, B. J. (2010). Hypoxic ischemia and proteasome dysfunction alter tau isoform ratio by inhibiting exon 10 splicing. Journal of Neurochemistry, 114(1), 160-170. https://doi.org/10.1111/j.1471-4159.2010.06732.x

Wang, Y., \& Mandelkow, E. (2015). Tau in physiology and pathology. Nature Reviews Neuroscience, 17, 22-35. https://doi.org/10.1038/nrn. 2015.1

Yrjänheikki, J., Koistinaho, J., Kettunen, M., Kauppinen, R. A., Appel, K., Hüll, M., \& Fiebich, B. L. (2005). Long-term protective effect of atorvastatin in permanent focal cerebral ischemia. Brain Research, 1052(2), 174-179.

Zhang, Q., Gao, T., Luo, Y., Chen, X., Gao, G., Gao, X., ... Dai, J. (2012). Transient focal cerebral ischemia/reperfusion induces early and chronic axonal changes in rats: Its importance for the risk of Alzheimer's disease. PLoS One, 7(3), e33722. https://doi.org/10.1371/ journal.pone.0033722

Zhang, R., Chopp, M., \& Zhang, Z. G. (2013). Oligodendrogenesis after cerebral ischemia. Frontiers in Cellular Neuroscience, 7(201). https://doi. org/10.3389/fncel.2013.00201

Zhang, Z. G., \& Chopp, M. (2009). Neurorestorative therapies for stroke: Underlying mechanisms and translation to the clinic. The Lancet Neurology, 8(5), 491-500. https://doi.org/10.1016/s1474-4422(09)70061-4

Zhao, Y., Wang, H., Chen, W., Chen, L., Liu, D., Wang, X., \& Wang, X. (2019). Melatonin attenuates white matter damage after focal brain ischemia in rats by regulating the TLR4/NF-KB pathway. Brain Research Bulletin, 150, 168-178. https://doi.org/10.1016/j.brainresbull.2019.05.019

\section{SUPPORTING INFORMATION}

Additional supporting information may be found online in the Supporting Information section at the end of this article.

How to cite this article: Villa González M, Vallés-Saiz L, Hernández IH, Avila J, Hernández F, Pérez-Alvarez MJ. Focal cerebral ischemia induces changes in oligodendrocytic tau isoforms in the damaged area. Glia. 2020;68:2471-2485. https://doi.org/10.1002/glia.23865 